\title{
Complete and competitive financial markets in a complex world
}

\section{Gianluca Cassese $^{1}$}

Received: 22 May 2020 / Accepted: 15 July 2021 / Published online: 17 September 2021

(c) The Author(s) 2021

\begin{abstract}
We investigate the possibility of completing financial markets in a model with no exogenous probability measure, with market imperfections and with an arbitrary sample space. We also consider whether such an extension may be possible in a competitive environment. Our conclusions highlight the economic role of complexity.
\end{abstract}

Keywords Competitive completion · Market completeness · Market power · No arbitrage $\cdot$ Sublinear pricing

Mathematics Subject Classification (2010) 91B24 · 91B08 · 60A99

JEL Classification $\mathrm{G} 10 \cdot \mathrm{G} 12$

\section{Introduction}

Since the seminal contributions of Arrow [4] and Radner [32], market completeness and the no-arbitrage principle have played a prominent rôle in financial economics. Market completeness, as first noted by Arrow, is a crucial property as it permits the optimal allocation of risk bearing among risk-averse agents. In fact, the equilibria of an economy under conditions of uncertainty but with competitive and complete financial markets are equivalent to those of an ordinary static economy so that classical welfare theorems apply. The equilibrium analysis on which this conclusion rests requires that financial markets are free of arbitrage opportunities. In the present paper, we consider the validity of these classical results in a complex world, that is, in the context of an economic model in which uncertainty is treated as a completely general and unrestricted phenomenon. As a general conclusion, we find that in a complex

I am grateful to the participants at SAET 2019 Conference, Ischia, for comments and suggestions.

G. Cassese

gianluca.cassese@unimib.it

1 Department of Economics, Statistics and Management, Università Milano-Bicocca, via Bicocca degli Arcimboldi 8, 20126 - Milan, Italy 
world, the interplay between uncertainty and asset prices is richer and more interesting than expected.

Indeed, the models of Arrow and Radner, and of much of the following literature on general equilibrium theory with financial markets, focus on an economy with a finite state space. This modelling choice is instrumental to and has its main advantage in the description of financial assets as contingent contracts, i.e., in purely economic terms. On the other hand, it has the drawback that this simplified representation of uncertainty makes it more challenging, in the absence of further elements, to justify market incompleteness. At the other extreme, in infinite-dimensional economic theory (see e.g. the review of Mas-Colell and Zame [31]), commodities and assets need to be defined as elements of some given space, the choice of which is most often a first step of crucial importance.

In the approach we propose hereafter, we retain Arrow's original idea that assets should be described solely in terms of the rights and obligations of the two counterparties on the occurrence of each future contingency and yet allow an arbitrary sample space $\Omega$. In particular, $\Omega$ is not endowed with any special structure, and the real-valued functions $X$ defined thereon, which describe assets' payoffs, need not be continuous or measurable in any specific sense. Following the thread of Cassese $[11,13]$, we do not even assume the existence of any exogenously given probability measure, a major difference with a large part of the financial literature as well as many important papers in equilibrium theory, such as Bewley [5]. Our starting point is rather a criterion of economic rationality which describes what all agents agree upon when saying that " $f$ is better than $g$ " (this modelling of rationality, first introduced in [13], is referred to as common order in Burzoni et al. [10]).

We believe that our general framework is indeed the natural setting for studying incomplete markets and for investigating the main aspects of a process of gradual completion of markets. In particular, we address the following questions: (a) Can an incomplete set of financial markets be extended to a complete one while preserving the basic economic principle of absence of arbitrage opportunities? (b) If so, can such an extension be supported by a competitive market mechanism?

Our finding is that neither question need have a positive answer. Concerning (a), we observe that the competition on financial markets may in principle produce two distinct outcomes. On the one hand, it lowers margins on currently traded assets and thus results in lower prices. On the other hand, competition involves the design and issuance of new securities. We argue that lower prices on the existing securities may destroy the possibility to obtain complete markets free of arbitrage opportunities in much the same way as predatory pricing in a monopolistic setting may prevent the entry of new, potential competitors. In general, the net effect of competition on collective welfare may be unclear. Regarding (b), we show that the completion of financial markets respecting the no-arbitrage principle may be not possible under linear pricing (which we take as synonymous to perfect competition). We actually provide an explicit example from which it clearly emerges that this negative conclusion has to do with the complexity of the economic environment described in the model. Indeed, most economic models treat economic complexity under probabilistic assumptions which do not permit a clear comprehension of this phenomenon. On the other hand, we show that if a no-arbitrage extension of markets with a limited degree of market power is possible, then markets admit a perfectly competitive extension as well. 
We should make clear that although it is indeed natural and appropriate on general grounds to interpret the extension of markets as the effect of financial innovation, an explicit model of the strategic behaviour of intermediaries, such as Allen and Gale [2] or Bisin [8], is beyond the scope of the present work. We rather study the properties of pricing functionals described as sublinear functionals on the space of traded assets' payoffs. The nonlinearity of prices captures the non-competitive nature of financial markets as well as the role of other market imperfections.

In recent years, there have been several papers in which the assumption of a given reference probability is relaxed, if not abandoned. Riedel [33] (and more recently Burzoni et al. [10]) suggests that an alternative approach to finance should be based on the concept of Knightian uncertainty. A typical implication of this approach is that a multiplicity of probability priors is given, rather than a single one. Some authors, including Bouchard and Nutz [9], interpret this multiplicity as an indication of model uncertainty, a situation in which each prior probability corresponds to a different model that possesses all the traditional properties but in which it is unknown which of the models should be considered the correct one. An exemplification is the paper by Epstein and Ji [22] in which model uncertainty translates into ambiguity concerning the volatility parameter. Other papers, among which are those by Davis and Hobson [17] and Acciaio et al. [1], take the sample space to consist of all trajectories of some underlying asset and study the prices of options written thereon based on a path-bypath or model-free definition of arbitrage.

In Sect. 2, we describe the model in all details, introduce the notion of arbitrage and prove some properties of prices. In Sect. 3, we characterise the set of pricing probability charges which is of crucial importance in our construction. In Sect. 4, we prove the first of our main results, Theorem 4.1, in which the possibility of completing markets is fully characterised. We show that in a complete financial market, although prices may in principle contain bubbles, there cannot be assets with a positive price but no intrinsic value. Theorem 4.1 provides an answer to question (a) above. In Sect. 5, we examine the viability of financial prices, which in our setting turns out to be stronger than the extension property. In Sect. 6, we establish a second fundamental result, Theorem 6.1, which answers question (b). It offers a complete characterisation of the existence of a fully competitive completion of financial markets. In particular, we prove that a competitive completion is possible if and only if one can obtain a completion in which the market power of intermediaries is limited. This result can also be read as a theoretical justification of the microstructure formula stating that asset prices are obtained by applying a spread on the asset fundamental value. Several additional implications are proved. We provide an explicit example of a financial market that admits no competitive completions as a consequence of a high degree of complexity. Given its importance in the reference literature, we examine in Sect. 7 the question of countable additivity and give necessary and sufficient conditions for the existence of a countably additive pricing probability.

\section{The economy}

We model the market as a triple $\left(\mathscr{X}, \geq_{*}, \pi\right)$, in which $\mathscr{X}$ describes the set of payoffs generated by the traded assets, $\geq_{*}$ the criterion of collective rationality used in the 
evaluation of investment projects, and $\pi$ is the price of each asset as a function of its payoff. Each of these elements is now described in detail.

Before getting to the model, we introduce some useful notation. Throughout, $\Omega$ will be an arbitrary nonempty set that we interpret as the sample space so that the family $\mathfrak{F}(\Omega)$ of real-valued functions on $\Omega$ is our ambient space; $\mathfrak{B}(\Omega)$ represents bounded functions. If $A \subseteq \mathfrak{F}(\Omega)$, we write $\bar{A}^{u}$ (resp. $\bar{A}^{\tau}$ ) to denote its closure in the topology of uniform distance (resp. in the topology $\tau$ ). If $\mathscr{A}$ is a $\sigma$-algebra of subsets of $\Omega, \mathfrak{F}(\Omega, \mathscr{A})$ denotes the family of $\mathscr{A}$-measurable functions, and we set $\mathfrak{B}(\Omega, \mathscr{A})=\mathfrak{F}(\Omega, \mathscr{A}) \cap \mathfrak{B}(\Omega)$. The symbol $b a(\mathscr{A})$ denotes the set of bounded, finitely additive set functions on $\mathscr{A}$, to which we refer as charges, while $\mathbb{P}_{b a}(\mathscr{A})$ designates the collection of probability charges, i.e., nonnegative charges with total mass 1 . We reserve the word probability with no further qualification and the symbol $\mathbb{P}(\mathscr{A})$ for classical (i.e., countably additive) probabilities. General references for the theory of charges and finitely additive integrals are Dunford and Schwartz [20, Chaps. III.2 and III.3] and Bhaskara Rao and Bhaskara Rao [6, Chap. 4].

\subsection{Economic rationality}

A natural order to assign to $\mathfrak{F}(\Omega)$ is the pointwise order, i.e., $f(\omega) \geq g(\omega)$ for all $\omega \in \Omega$, also written as $f \geq g$. The lattice symbols $|f|$ or $f^{+}$always refer to that order.

Natural as it may appear, the pointwise order is not an adequate description of how economic agents rank random quantities save when the underlying sample space is particularly simple, such as a finite set. For example, it is well documented that investors base their decisions on a rather incomplete assessment of the potential losses arising from the selected portfolios, exhibiting a sort of asymmetric attention that leads them to neglect some scenarios, in contrast with a pointwise ranking of investment projects (see Cassese [11] for a short discussion of some inattention phenomena relevant for financial decisions). In a complex world, in which the attempt to formulate a detailed description of $\Omega$ is out of reach, rational inattention is just one possible approach to deal with complexity, possibly not too different from the restriction to measurable quantities adopted by classical probability.

In this paper, following the ideas of Cassese [13], we treat monotonicity as a primitive economic notion represented by a further transitive, reflexive binary relation on $\mathfrak{F}(\Omega)$. To distinguish it from the pointwise order $\geq$, we use the symbol $\geq_{*}$ (the asymmetric part of $\geq_{*}$ will be written as $>_{*}$ ).

We assume the following properties:

$$
\begin{aligned}
& \text { (i) } f \geq g \text { implies } f \geq_{*} g \quad \text { and } \quad \text { (ii) } t \in \mathbb{R}_{++} \text {implies } f+t>_{*} f \text {; } \\
& \text { if } f \geq_{*} g \text {, then } \lambda f+h \geq_{*} \lambda g+h, \quad \lambda \in \mathfrak{B}_{+}(\Omega), h \in \mathfrak{F}(\Omega) \text {; } \\
& \text { if } f>_{*} 0 \text {, then } f \wedge 1>_{*} 0 .
\end{aligned}
$$

These will be the basis for what follows. The first paper to treat monotonicity in an axiomatic way was of course Kreps [28]. In [13], axioms similar to (2.1)-(2.3) were first introduced and it was shown that a ranking with such properties may equivalently be deduced from a cash-additive risk measure. A very similar approach to ours has later been adopted in the recent paper by Burzoni et al. [10]. 
Of course, the symmetric part of $\geq_{*}$ induces a corresponding equivalence relation $\sim_{*}$. It is useful to remark that if $f \geq_{*} 0$, then by property (2.2), $f \mathbb{1}_{\{f \leq 0\}} \geq_{*} 0$ so that $f \geq_{*} f-2 f \mathbb{1}_{\{f \leq 0\}}=|f|$, i.e., $|f| \sim_{*} f$. Based on $\sim_{*}$, we can also construct the collection of negligible sets

$$
\mathscr{N}_{*}=\left\{A \subseteq \Omega: 0 \sim_{*} \mathbb{1}_{A}\right\},
$$

and if $\mathscr{A}$ is an arbitrary $\sigma$-algebra of subsets of $\Omega$ which contains $\mathscr{N}_{*}$, the subset $\mathbb{P}_{b a}\left(\mathscr{A}, \mathscr{N}_{*}\right)$ consisting of probability charges on $\mathscr{A}$ which vanish on $\mathscr{N}_{*}$. Every subset of $\Omega$ not included in $\mathscr{N}_{*}$ is called non-negligible. It will be useful to define the space

$$
\mathfrak{B}\left(\Omega, \mathscr{A}, \mathscr{N}_{*}\right)=\left\{f \in \mathfrak{F}(\Omega): f \sim_{*} b \text { for some } b \in \mathfrak{B}(\Omega, \mathscr{A})\right\} .
$$

We note that any exogenously given probability charge $P \in \mathbb{P}_{b a}(\mathscr{A})$ on some $\sigma$-algebra $\mathscr{A}$ (countably or finitely additive) induces a corresponding ranking defined as

$$
f \geq P g \quad \text { if and only if } \quad \sup _{\varepsilon>0} P[f \leq g-\varepsilon]=0, \quad f, g \in \mathfrak{F}(\Omega, \mathscr{A}),
$$

which satisfies the above axioms (2.1)-(2.3). In this case, a set is negligible if and only if it is $P$-null. Clearly, if $P$ is a probability, the ranking $\geq_{P}$ is just the $P$-a.s. ranking. The same construction may be extended by replacing $P$ with a family $\mathscr{P} \subseteq \mathbb{P}_{b a}(\mathscr{A})$ of probability charges and defining accordingly

$$
f \geq \mathscr{P} h \quad \text { if and only if } \quad f \geq_{P} h \text { for all } P \in \mathscr{P} .
$$

The ranking $\geq \mathscr{P}$ defined in (2.5) arises in connection with the model uncertainty approach mentioned in the introduction and exemplified by the paper by Bouchard and Nutz [9]. In this approach, each element $P$ of the given collection $\mathscr{P}$ is a model. It should be noted that the choice of Bouchard and Nutz to take $\mathscr{P}$ to be a set of probabilities has considerable implications on $\mathscr{N}_{*}$ which coincides with the collection of sets which are $P$-null for every $P \in \mathscr{P}$ and is therefore closed with respect to countable unions. We return to the ranking $\geq \mathscr{P}$ in Example 6.3.

We next provide some concrete examples of the partial order $\geq_{*}$ that arise from decision theory.

Example 2.1 Let $\succeq_{\alpha}$ represent the preference system of agent $\alpha$ defined over the whole of $\mathfrak{F}(\Omega)$. Assume that $\succeq_{\alpha}$ satisfies the following monotonicity properties valid for all $f, g \in \mathfrak{F}(\Omega)$ :

$$
\text { (i) } f \geq g \text { implies } f \succeq_{\alpha} g \quad \text { and } \quad \text { (ii) } t \in \mathbb{R}_{++} \text {implies } f+t \succ_{\alpha} f \text {. }
$$

These assumptions are indeed minimal in economic models with an infinite-dimensional commodity space; see e.g. Bewley [5, Theorem 1] or Mas-Colell [30, p. 1041]. We may deduce an implicit, subjective criterion $\geq_{\alpha}$ of monotonicity (or rationality) by letting

$$
f \geq_{\alpha} g \quad \text { if and only if } \quad \lambda(f-g)+h \succeq_{\alpha} h, \quad \lambda, h \in \mathfrak{F}(\Omega), \lambda \geq 0 .
$$


A mathematical criterion $\geq_{*}$ describing collective rationality may then be defined as the meet of all such individual rankings, i.e., as

$$
f \geq_{*} g \quad \text { if and only if } \quad f \geq_{\alpha} g \text { for each agent } \alpha \in \mathfrak{A} \text {. }
$$

Notice that in this case, $f>_{*} g$ means that all agents agree on $f \geq_{\alpha} g$ and there exists at least one agent $\alpha_{0} \in \mathfrak{A}$ according to which $f>_{\alpha_{0}} g$.

Example 2.2 Let an agent $\alpha$ have expected utility preferences $U_{\alpha}(f)=\int u_{\alpha}(f) d P_{\alpha}$ with a monotonic increasing utility function $u_{\alpha}$ satisfying $u_{\alpha}(t)>u_{\alpha}(0)=0$ for each $t>0$. Define $\geq_{\alpha}$ as in Example 2.1. Then $f \geq_{\alpha} g$ implies by (2.6) that

$$
U_{\alpha}(0) \geq U_{\alpha}\left((f-g+t) \mathbb{1}_{\{f-g<-t\}}\right) \geq U_{\alpha}\left(t \mathbb{1}_{\{f-g<-t\}}\right)=u_{\alpha}(t) P_{\alpha}[f-g<-t,]
$$

i.e., that $f \geq P_{\alpha} g$. The converse is of course also true. Thus $\geq_{\alpha}$ coincides with $\geq_{P_{\alpha}}$. If we aggregate the $\geq_{\alpha}$ rankings via the unanimity rule (2.7), we obtain thus the ranking $\geq \mathscr{P}$ defined in (2.5), with $\mathscr{P}=\left\{P_{\alpha}: \alpha \in \mathfrak{A}\right\}$.

We return to Example 2.2 in Example 6.3.

Example 2.3 In the theory of decision making, several models, starting with Schmeidler [34], have treated the problem of choice under uncertainty by means of set functions with weak properties, often not even additive. Fix $v: 2^{\Omega} \rightarrow[0,1]$ and define $\geq_{v}$ as in (2.4). Then $\geq_{v}$ satisfies properties (2.1)-(2.3) if and only if (i) $v[\varnothing]=0$ and (ii) $v[A] \leq v[B]$ when $A \subseteq B$, i.e., when $v$ is a capacity. Nevertheless, the binary relation $\geq_{v}$ (which is always reflexive by virtue of (i)) is transitive if and only if $v$ satisfies in addition the further property

$$
\nu[A \cup B]=v[A], \quad B \subseteq \Omega, v[B]=0,
$$

which is sometimes referred to as null-additivity. A strongly subadditive (or submodular) capacity, see Dellacherie and Meyer [19, III.30], clearly possesses all the above properties, while a supermodular one need not satisfy (2.8).

The conclusion of the preceding Example 2.3 is given a formal statement in the following result.

Lemma 2.4 A ranking that satisfies properties (2.1)-(2.3) originates from a set function $v: 2^{\Omega} \rightarrow[0,1]$ via (2.4) if and only if $v$ is a null-additive capacity.

Example 2.5 In behavioural economics and experimental psychology, it is often argued that decisions are taken by focusing attention on a restricted class of possible scenarios to which agents attach special importance or simply over which they feel relatively confident or just more informed. Let $\mathscr{F}$ be a family of nonempty subsets of $\Omega$, representing the sets relatively to which the agent is fully confident. An agent may then rank alternatives in accordance with the criterion

$$
f \geq \mathscr{F} h \quad \text { if and only if } \quad\{f-h>-t\} \in \mathscr{F}, t>0 .
$$


Again, one easily concludes that $\geq \mathscr{F}$ possesses the properties (2.1)-(2.3) if and only if $\mathscr{F}$ is a filter of subsets of $\Omega$. In accordance with Dunford and Schwartz [20, I.7.11], let $\mathscr{F}_{*}$ be some ultrafilter refining $\mathscr{F}$ and define $P \in \mathbb{P}_{b a}\left(2^{\Omega}\right)$ by letting $P[G]=1$ if $G \in \mathscr{F}_{*}$ and $P[G]=0$ otherwise. Then $f \geq \mathscr{F} h$ implies $f \geq_{P} h$; the converse implication, however, need not be true.

An interesting question raised by the preceding examples concerns the conditions under which a given ranking $\geq_{*}$ coincides with the ranking $\geq_{P}$ for some endogenous probability charge $P$. Notice that in Example 2.5, we only showed that $\geq_{P}$ is compatible with $\geq \mathscr{F}$, but we may still have that $f \geq_{P} h$ while $f \geq \mathscr{F} h$. We obtain an indirect answer to this question in Theorem 6.5.

\subsection{Assets}

We posit the existence of an asset whose final payoff and current price are used as numéraire of the payoff and price of all other assets, respectively. Each existing asset is assumed to be traded on the market, is identified with its payoff expressed in units of the numéraire and modelled as an element of $\mathfrak{F}(\Omega)$. The market is then a convex set $\mathscr{X} \subseteq \mathfrak{F}(\Omega)$ containing the origin as well as the function identically equal to 1 (simply indicated by 1 ). Notice that similarly to Jouini and Kallal [27], we do not assume that investments may be replicated on any arbitrary scale, i.e., that $\mathscr{X}$ is a convex cone, as is customary in this literature. Restrictions to the investment scale are commonly met on the market whenever investors are subject to margin requirements or the provision of a collateral.

We assume in addition that each $X \in \mathscr{X}$ satisfies $X \geq_{*} a$ for some $a \in \mathbb{R}$ and that

$$
X+\lambda \in \mathscr{X} \quad \text { for all } X \in \mathscr{X}, \lambda \geq 0 \text {. }
$$

The first of these assumptions constrains the assets traded on the market to bear a limited risk of losses and may be interpreted as a restriction imposed by some regulator; the second permits agents, which in principle may only form convex portfolios, to invest into the numéraire asset an unlimited amount of capital. Notice that since the numéraire cannot be shorted, the construction of zero cost portfolios - or selffinancing strategies - need not be possible (outside of the trivial case of assets with a negative price which are self-financed by definition). We do not assume that the market prohibits short positions, but rather that in the presence of credit risk, long and short positions, even if permitted, should be regarded as two different investments as they bear potentially different levels of risk. In other words, when taking short positions, investors affect the implicit counterparty risk and modify de facto the final payoff of the shorted asset.

\subsubsection{Market extensions}

The issuance of new securities may result in an extension of the set $\mathscr{X}$ of traded assets and possibly in the completion of the existing markets. Let us mention that in traditional models, in which asset payoffs are modelled as elements of an appropriate ambient space - often a Lebesgue space $L^{p}(\Omega, \mathscr{F}, P)$-, markets are considered to 
be complete if each elements of that space is attained by some traded asset. In our setting, defining market completeness is more delicate. The idea that all elements of $\mathfrak{F}(\Omega)$ may be traded is indeed too ambitious, as it would be difficult to define a price functional on such a large domain.

In order to have an appropriate notion of completeness of markets, we find it convenient to introduce a $\sigma$-algebra $\mathscr{A}$ of subsets of $\Omega$ satisfying

$$
\bigcup_{\left\{f \sim_{*} X: X \in \mathscr{X}\right\}} \sigma(f) \subseteq \mathscr{A}
$$

and to define

$$
L(\mathscr{X}, \mathscr{A})=\left\{g \in \mathfrak{F}(\Omega, \mathscr{A}): \lambda X \geq_{*}|g| \text { for some } \lambda>0, X \in \mathscr{X}\right\},
$$

which may be loosely interpreted as the set of ( $\mathscr{A}$-measurable) superhedgeable claims. We then define markets to be complete relatively to $\mathscr{A}$ (or $\mathscr{A}$-complete, for brevity) whenever each element of $L(\mathscr{X}, \mathscr{A})$ is attained by a corresponding asset. In other words, we define market completeness conditionally on the $\sigma$-algebra $\mathscr{A}$, and of course a whole hierarchy of completions is possible as $\mathscr{A}$ ranges between the two extrema of the minimal $\sigma$-algebra $\mathscr{A}_{0}$ generated by the elements equivalent to some $X \in \mathscr{X}$ and the power set of $\Omega$. The introduction of a measurable structure, which implies no restriction in our analysis, serves two distinct purposes. On the one hand, it makes the comparison with the traditional literature fully transparent; on the other hand, it permits putting the extension problem in a clearer relation with the notion of complexity that we identify with the fineness of $\mathscr{A}$. A fully complex model corresponds then to the case in which $\mathscr{A}$ is the power set of $\Omega$, a case perfectly possible in our setting.

Lemma 2.6 The set $L(\mathscr{X}, \mathscr{A})$ defined in (2.9) is a vector sublattice of $\mathfrak{F}(\Omega, \mathscr{A})$ containing $\mathscr{X}$ and $\mathfrak{B}\left(\Omega, \mathscr{A}, \mathscr{N}_{*}\right)$.

Proof The last inclusion is clear from the definition of $L(\mathscr{X}, \mathscr{A})$. It is easily seen that $g \in L(\mathscr{X}, \mathscr{A})$ if and only if $|g| \in L(\mathscr{X}, \mathscr{A})$; moreover, if $\lambda_{i} X_{i} \geq_{*}\left|g_{i}\right|$ and $a_{i} \in \mathbb{R}$ for $i=1,2$, then letting $\lambda=\left|a_{1}\right| \lambda_{1}+\left|a_{2}\right| \lambda_{2}$ and $X=X_{1}\left(\left|a_{1}\right| \lambda_{1} / \lambda\right)+X_{2}\left(\left|a_{2}\right| \lambda_{2} / \lambda\right)$, we conclude from (2.2) that

$$
\lambda X=\left|a_{1}\right| \lambda_{1} X_{1}+\left|a_{2}\right| \lambda_{2} X_{2} \geq_{*}\left|a_{1}\right|\left|g_{1}\right|+\left|a_{2}\right|\left|g_{2}\right| \geq\left|a_{1} g_{1}+a_{2} g_{2}\right|,
$$

so that $g_{1}, g_{2} \in L(\mathscr{X}, \mathscr{A})$ and $a_{1}, a_{2} \in \mathbb{R}$ imply $a_{1} g_{1}+a_{2} g_{2} \in L(\mathscr{X}, \mathscr{A})$. This proves that $L(\mathscr{X}, \mathscr{A})$ is a vector sublattice of $\mathfrak{F}(\Omega, \mathscr{A})$. If $Y \in \mathscr{X}$, then $Y+a \geq_{*} 0$ for some $a \in \mathbb{R}_{+}$so that $Y+2 a \in \mathscr{X}$ and $Y+2 a \geq_{*}|Y+a|+a \geq|Y|$ so that $Y \in L(\mathscr{X}, \mathscr{A})$.

When markets are $\mathscr{A}$-complete, each element $f \in \mathfrak{B}\left(\Omega, \mathscr{A}, \mathscr{N}_{*}\right)$ corresponds to the payoff of some asset traded on the market. In the special case in which $\geq_{*}$ is generated by some $P \in \mathbb{P}(\mathscr{A})$, market completeness implies that all claims with payoff in $L^{\infty}(\Omega, \mathscr{A}, P)$ are attained. 


\subsection{Prices}

In financial markets with frictions and limitations to trade, normalised prices are best modelled as positively homogeneous, subadditive functionals $\pi: \mathscr{X} \rightarrow \mathbb{R}$ of the asset payoff, satisfying $\pi(1)=1$, the monotonicity condition

$$
X, Y \in \mathscr{X} \text { and } X \geq_{*} Y \quad \text { imply } \quad \pi(X) \geq \pi(Y)
$$

and cash-additivity

$$
\pi(X+a)=\pi(X)+a \quad \text { for all } X \in \mathscr{X}, a \in \mathbb{R} \text { such that } X+a \in \mathscr{X} \text {. }
$$

This latter property, defined in slightly different terms, is discussed at length relatively to risk measures in El Karoui and Ravanelli [21]. In the context of nonlinear pricing, cash-additivity is virtually always assumed in a much stronger version, namely for all $X \in \mathscr{X}$ and all $a \in \mathbb{R}$; see e.g. Araujo et al. [3, Definition 1].

The nonlinearity of financial prices is a well-known empirical feature documented in the microstructure literature (see e.g. the exhaustive survey by Biais et al. [7]) and essentially accounts for the auxiliary services that are purchased when investing in an asset, such as liquidity provision and inventory services. Subadditivity captures the idea that these services are imperfectly divisible and that they are supplied in conditions of limited competition. The degree of nonlinearity of prices, computed as

$$
\mathfrak{m}(\pi)=\sup _{f_{1}, \ldots, f_{N} \in \mathfrak{B}_{+}\left(\Omega, \mathscr{A}, \mathscr{N}_{*}\right) \cap \mathscr{X}} \frac{\sum_{i \leq N} \pi\left(f_{i}\right)-\pi\left(\sum_{i \leq N} f_{i}\right)}{\sum_{i \leq N} \pi\left(f_{i}\right)},
$$

plays a major role in Sect. 6 and measures the lack of competitiveness on financial markets. Although deviations from the competitive paradigm may originate from several sources, we find it convenient to interpret the polar cases $\mathfrak{m}(\pi)=0$ and $\mathfrak{m}(\pi)=1$ as indication of perfect competition and of full monopoly power, respectively.

Cash-additivity implicitly assumes that the numéraire is traded and priced on a separate market - as is normally the case with treasury bonds, but not necessarily with other assets. Concerning positive homogeneity, this property is consistent with market imperfections such as the bid-ask spread, but contrasts in general with fixed costs. In Jouini and Kallal [27], prices are described by a convex functional, thus are not necessarily positively homogeneous (however, in [27, Assumption 2.1], the assumption of monotonicity of prices is omitted). Indeed, a number of our results still hold true even for convex price functionals; however, if the price functional is not positively homogeneous, the superhedging duality (3.2) established below in Theorem 3.1 is no longer true in general.

We also require that prices be free of arbitrage opportunities, a property which we define as

$$
X \in \mathscr{X} \text { and } X>_{*} 0 \text { imply } \pi(X)>0 .
$$

See Cassese [13] for a short discussion of alternative definitions of arbitrage in an imperfect market. We do not adopt the pointwise definition of arbitrage suggested 
e.g. in Acciaio et al. [1], as this would implicitly correspond to assuming a form of rationality on economic agents even more extreme than probabilistic sophistication.

Of course, (2.12) implies that $\pi(X) \geq 0$ whenever $X \geq_{*} 0$, while (2.10) need not follow from (2.12) if short selling is not permitted. We notice that the situation $X>_{*} \pi(X)>0$, though clearly exceptional, does not represent in our model an arbitrage opportunity because of the potential infeasibility of short positions in the numéraire asset. A firm experiencing difficulties in raising funds for its projects and competing with other firms in a similar position may offer abnormally high returns to induce investors to purchase its debt.

A functional satisfying all the preceding properties - including (2.12) - is called a price functional, and the corresponding set is indicated with the symbol $\Pi(\mathscr{X})$. We thus agree that market prices are free of arbitrage by definition and avoid recalling this crucial property. At times, though, it is mathematically useful to consider pricing functionals for which cash-additivity and/or the no-arbitrage property (2.12) may fail. These are denoted by the symbol $\Pi_{0}(\mathscr{X})$. We note that even if $\pi_{0} \in \Pi_{0}(\mathscr{X})$ fails to be cash-additive, it always has a cash-additive part $\pi_{0}^{a}$, namely the functional

$$
\pi_{0}^{a}(X)=\inf \left\{\pi_{0}(X+t)-t: t \in \mathbb{R} \text { such that } X+t \in \mathscr{X}\right\}, \quad X \in \mathscr{X} .
$$

It is routine to show that $\pi_{0}^{a}$ is the greatest cash-additive element dominated by $\pi_{0}$.

Example 2.7 In classical continuous-time financial models, the discounted final payoff of each investment takes the form

$$
X^{w, \theta}=w+\int_{0}^{\infty} \theta_{u} d S_{u}
$$

in which $w$ is the initial wealth invested, the discounted price process $S$ is a semimartingale on some filtered probability space and $\theta$ is an element of a suitably defined set $\Theta$ of admissible trading strategies for which $\int_{0}^{\infty} \theta_{u} d S_{u}=\lim _{t \rightarrow \infty} \int_{0}^{t} \theta_{u} d S_{u}$ exists a.s. Assuming that each $\theta \in \Theta$ is maximal, in the sense that $X^{w^{\prime}, \theta^{\prime}} \geq X^{w, \theta}$ a.s. for some $w^{\prime}$ and $\theta^{\prime} \in \Theta$ implies $w^{\prime} \geq w$, then $\pi\left(X^{w, \theta}\right)=w$. Given the possibility of shorting the numéraire, each investment is naturally associated with a corresponding self-financing strategy with final payoff $X^{0, \theta}=\int_{0}^{\infty} \theta_{u} d S_{u}$. The no-arbitrage condition (2.12) restricted to self-financing strategies implies then that no such strategy may produce a strictly positive final payoff, i.e., that

$$
\left\{X^{0, \theta}: \theta \in \Theta\right\} \cap L^{0}(\Omega, \mathscr{A}, P)_{++}=\varnothing .
$$

Compare with Delbaen and Schachermayer [18, Definition 2.8(i)].

\section{Pricing charges}

Associated with each price $\pi \in \Pi(\mathscr{X})$ is the convex cone

$$
\mathscr{C}(\pi, \mathscr{A})=\left\{g \in \mathfrak{F}(\Omega, \mathscr{A}): \lambda(X-\pi(X)) \geq_{*} g \text { for some } \lambda>0 \text { and } X \in \mathscr{X}\right\},
$$


and, more importantly, the collection of pricing probability charges, namely

$$
\begin{aligned}
& \mathscr{M}_{0}(\pi, \mathscr{A})=\left\{m \in \mathbb{P}_{b a}(\mathscr{A}): L(\mathscr{X}, \mathscr{A}) \subseteq L^{1}(m)\right. \text { and } \\
& \left.\qquad \pi(X) \geq \int X d m \text { for every } X \in \mathscr{X}\right\} .
\end{aligned}
$$

In Cassese [13], we used the term pricing measure to define a positive charge dominated by $\pi$ without restricting it to be a probability charge. The focus on probability charges will be clear after Theorem 4.1. A definition of the finitely additive integral and its properties may be found in Dunford and Schwartz [20, III.2.17].

The following basic result illustrates the role of cash-additivity and of the set $\mathscr{M}_{0}(\pi, \mathscr{A})$. In particular, property (3.2) may be considered as our version of the classical superhedging duality theorem. The first part of Theorem 3.1 is mainly a consequence of the Hahn-Banach theorem and is true even if the price functional $\pi_{0}$ is just convex. The superhedging formula (3.2), on the other hand, necessarily requires positive homogeneity.

Theorem 3.1 For given $\pi_{0} \in \Pi_{0}(\mathscr{X})$, the set $\mathscr{M}_{0}(\pi, \mathscr{A})$ is nonempty and each $m \in \mathscr{M}_{0}(\pi, \mathscr{A})$ satisfies

$$
\int f d m \geq \int g d m \quad \text { for all } f, g \in L(\mathscr{X}, \mathscr{A}) \text { with } f \geq_{*} g .
$$

Moreover, $\mathscr{M}_{0}\left(\pi_{0}, \mathscr{A}\right)=\mathscr{M}_{0}\left(\pi_{0}^{a}, \mathscr{A}\right)$ and

$$
\pi_{0}^{a}(X)=\sup _{m \in \mathscr{M}_{0}\left(\pi_{0}, \mathscr{A}\right)} \int X d m, \quad X \in \mathscr{X} \cap \mathfrak{B}(\Omega, \mathscr{A}) .
$$

Finally, the set $\mathscr{M}_{0}\left(\pi_{0}, \mathscr{A}\right)$ is convex and compact in the weak* topology of ba $(\mathscr{A})$ (i.e., the topology induced by $\mathfrak{B}(\Omega, \mathscr{A})$ on ba $(\mathscr{A})$ ).

Proof We simply use Hahn-Banach theorem and the integral representation of a positive linear functional $\phi$ on the vector lattice $L(\mathscr{X}, \mathscr{A})$ as

$$
\phi(f)=\phi^{\perp}(f)+\int f d m_{\phi}, \quad f \in L(\mathscr{X}, \mathscr{A}),
$$

established in Theorem A.3. In (3.3), $m_{\phi}$ is a positive charge on $\mathscr{A}$ such that we have $L(\mathscr{X}, \mathscr{A}) \subseteq L^{1}\left(\Omega, \mathscr{A}, m_{\phi}\right) ;$ moreover, $m_{\phi} \in \mathbb{P}_{b a}(\mathscr{A})$ if and only if $\phi(1)=1$.

We easily deduce from (2.10) that the functional defined by

$$
\bar{\pi}_{0}(g)=\inf \left\{\lambda \pi_{0}(X): \lambda>0, X \in \mathscr{X}, \lambda X \geq_{*} g\right\}, \quad g \in L(\mathscr{X}, \mathscr{A}),
$$

is an element of $\Pi_{0}(L(\mathscr{X}, \mathscr{A}))$ which extends $\pi_{0}$. By Hahn-Banach theorem, we can find a linear functional $\phi$ on $L(\mathscr{X}, \mathscr{A})$ such that $\phi \leq \bar{\pi}_{0}$ and $\phi(1)=1$. Necessarily, $f \geq_{*} 0$ implies $\phi(f) \geq 0$ so that by (2.2), $\phi$ is $\geq_{*}$-monotone and thus 
$m_{\phi} \in \mathbb{P}_{b a}\left(\mathscr{A}, \mathscr{N}_{*}\right)$. To show that $m_{\phi} \in \mathscr{M}_{0}\left(\pi_{0}, \mathscr{A}\right)$, observe that each $X \in \mathscr{X}$ admits $a \in \mathbb{R}$ such that $X \geq_{*} a$ so that we have $\phi^{\perp}(X) \geq \phi^{\perp}(a)=0$ and therefore $\pi_{0}(X)=\bar{\pi}_{0}(X) \geq \phi(X) \geq \int X d m_{\phi}$. Suppose now that $m \in \mathscr{M}_{0}\left(\pi_{0}, \mathscr{A}\right)$ and that $f, g \in L(\mathscr{X}, \mathscr{A})$ and $f \geq_{*} g$. Then by $(2.2),\{f-g \leq-\varepsilon\} \in \mathscr{N}_{*}$ for all $\varepsilon>0$ so that

$$
\int(f-g) d m=\int_{\{f-g>-\varepsilon\}}(f-g) d m \geq-\varepsilon .
$$

We deduce (3.1) from $f, g \in L^{1}(\Omega, \mathscr{A}, m)$.

Concerning the claim $\mathscr{M}_{0}\left(\pi_{0}, \mathscr{A}\right)=\mathscr{M}_{0}\left(\pi_{0}^{a}, \mathscr{A}\right)$, it is clear that the inequality $\pi_{0}^{a} \leq \pi_{0}$ induces the inclusion $\mathscr{M}_{0}\left(\pi_{0}^{a}, \mathscr{A}\right) \subseteq \mathscr{M}_{0}\left(\pi_{0}, \mathscr{A}\right)$. To prove the converse, choose $m \in \mathscr{M}_{0}\left(\pi_{0}, \mathscr{A}\right)$. If $X \in \mathscr{X}$ and $t \in \mathbb{R}$ are such that $X+t \in \mathscr{X}$, then

$$
\pi_{0}(X+t)-t \geq \int(X+t) d m-t=\int X d m
$$

so that $m \in \mathscr{M}_{0}\left(\pi_{0}^{a}, \mathscr{A}\right)$.

The cash-additive part $\bar{\pi}_{0}^{a}$ of $\bar{\pi}_{0}$, obtained as in (2.13), is easily seen to be an extension of $\pi_{0}^{a}$ to $L(\mathscr{X}, \mathscr{A})$. Of course, $\bar{\pi}_{0}^{a}$ is the pointwise supremum of the linear functionals $\phi$ that it dominates so that (3.2) follows if we show that $m_{\phi} \in \mathbb{P}_{b a}(\mathscr{A})$ for all such $\phi$. But this is clear since $\bar{\pi}_{0}^{a} \geq \phi$ implies that $\phi$ is positive on $L(\mathscr{X}, \mathscr{A})$. Moreover,

$$
\bar{\pi}_{0}^{a}(f)=\bar{\pi}_{0}^{a}(f+t)-t \geq \phi(f+t)-t=\phi(f)-t(1-\phi(1)), \quad t \in \mathbb{R},
$$

which contradicts the inequality $\bar{\pi}_{0}^{a} \geq \phi$ unless $\phi(1)=1$ and thus $m_{\phi} \in \mathbb{P}_{b a}(\mathscr{A})$.

The last claim is an obvious implication of the Tychonoff theorem; see Dunford and Schwartz [20, I.8.5].

Pricing probability charges closely correspond to the risk-neutral measures which are ubiquitous in the traditional financial literature since the seminal paper of Harrison and Kreps [24]. We only highlight that the existence of pricing charges and their properties are entirely endogenous here and do not depend on any special mathematical assumption - and actually not even on the absence of arbitrage. Nevertheless, Theorem 3.1 relies on property (2.10) which may be seen as a weaker form of the no-arbitrage property.

In traditional models, the condition $\mathscr{M}_{0}(\pi, \mathscr{A}) \neq \varnothing$ is obtained via the Riesz representation theorem (here replaced with Theorem A.3) and requires an appropriate topological structure. Finite additivity is a direct consequence of our minimal approach. The view expressed by Bewley [5, p. 516] that charges have "no economic interpretation" and the elements he offers in favour of the choice of the Mackey topology only make sense if a reference, countably additive measure is assumed to be given exogenously. It is, in other words, a somewhat circular argument. We argue in Sect. 7 that in a model treating economic rationality as a primitive concept, the economic role of countable additivity is far from clear.

It is customary to interpret the expected value $\int X d m$ of the asset payoff as its fundamental value, although the value so obtained may vary significantly as $m$ ranges 
across the set of pricing charges. The intrinsic value of the asset, computed as

$$
\sup _{m \in \mathscr{M}_{0}(\pi, \mathscr{A})} \int X d m
$$

corresponds to the most optimistic among such evaluations. A bubble is customarily defined as the spread between the price of an asset and its intrinsic value, so that in our model it is computed as

$$
\beta_{\pi}(X):=\pi(X)-\sup _{m \in \mathscr{M}_{0}(\pi, \mathscr{A})} \int X d m .
$$

In a recent paper, Herdegen and Schweizer [25, Sect. 6] introduce the notion of a strong bubble which is somehow similar to ours; it makes use of superreplication prices to compute the fundamental value of an asset so that the existence of a bubble is independent of the choice of the numéraire. According to Theorem 3.1, assets with a bounded payoff admit no bubbles. At present, however, we cannot exclude the extreme situation of a pure bubble, i.e., of an asset $X \in \mathscr{X}$ such that $X \geq_{*} 0$,

$$
\pi(X)>0, \quad \text { but } \quad \sup _{m \in \mathscr{M}_{0}(\pi, \mathscr{A})} \int X d m=0 .
$$

In Theorem 4.1, we obtain among other things a full characterisation of pure bubbles.

At this level of generality, we cannot conclude in favour of the pricing formula popular in microstructure models and according to which asset prices are obtained by applying some spread to its fundamental value, such as

$$
\pi(X)=(1+\alpha(X)) \int X d m \quad \text { with }|\alpha(X)|<1, X \in \mathscr{X} .
$$

We easily see that (3.6) is a stronger condition than the absence of pure bubbles. This formula is discussed again in Sect. 6.

We close by remarking that each $m \in \mathscr{M}_{0}(\pi, \mathscr{A})$ induces a linear functional on $L(\mathscr{X}, \mathscr{A})$. The locally convex linear topology induced by these functionals and denoted by $\tau(\pi)$ is weaker than the classical weak topology.

\section{Market completeness}

Competition among financial intermediaries may involve existing assets and/or the launch of new financial claims. As a consequence, it may produce two different effects: (a) a reduction of intermediation margins, and thus lower asset prices, and (b) an enlargement of the set $\mathscr{X}$ of traded assets, thus contributing to completing the markets. This short discussion justifies our interest in the set

$$
\operatorname{Ext}(\pi, \mathscr{A})=\left\{\pi^{\prime} \in \Pi(L(\mathscr{X}, \mathscr{A})):\left.\pi^{\prime}\right|_{\mathscr{X}} \leq \pi\right\} .
$$

The set $\operatorname{Ext}_{0}(\pi, \mathscr{A})$ is defined likewise, but with $\Pi(\mathscr{X})$ replaced with $\Pi_{0}(\mathscr{X})$. Recall that $\Pi(\mathscr{X})$ and $\Pi_{0}(\mathscr{X})$ are introduced just before (2.13). 
In this section, we want to address the following question: Under what conditions is it possible to extend the actual markets to obtain an economy with complete financial markets without violating the no-arbitrage principle? This translates into the mathematical condition $\operatorname{Ext}(\pi, \mathscr{A}) \neq \varnothing$ and, if $\pi^{\prime} \in \operatorname{Ext}(\pi, \mathscr{A})$, we speak of $\left(L(\mathscr{X}, \mathscr{A}), \geq_{*}, \pi^{\prime}\right)$ as an $\mathscr{A}$-completion of $\left(\mathscr{X}, \geq_{*}, \pi\right)$. See the discussion on completeness before Lemma 2.6, and note that any (traded) asset must have a price.

The first papers to focus on the extension property of financial prices were those by Harrison and Kreps [24] and Kreps [28] (see also Cassese [11, Theorem 8.1]), although this aspect has later been somehow neglected in the following literature. In their approach, the completion property is related to (and in fact in most cases equivalent to) the concept of viability that we discuss later. Recalling the discussion in Sect. 2.2.1, we remark that our definition of $\mathscr{A}$-completion does not preclude that markets may in principle be further extended, e.g. by passing to a larger $\sigma$-algebra than $\mathscr{A}$. However, given that $\mathscr{A}$ is quite arbitrary, our results carry over very simply.

We obtain the following complete characterisation for the case of cash-additive $\mathscr{A}$-completions.

Theorem 4.1 For a market $\left(\mathscr{X}, \geq_{*}, \pi\right)$, the following properties are equivalent:

(a) $\pi$ satisfies the condition

$$
\overline{\mathscr{C}(\pi, \mathscr{A})}^{\tau(\pi)} \cap\left\{f \in \mathfrak{F}(\Omega, \mathscr{A}): f>_{*} 0\right\}=\varnothing .
$$

(b) $\pi$ satisfies the condition

$$
\overline{\mathscr{C}(\pi, \mathscr{A})}^{u} \cap\left\{f \in \mathfrak{F}(\Omega, \mathscr{A}): f>_{*} 0\right\}=\varnothing .
$$

(c) The market $\left(\mathscr{X}, \geq_{*}, \pi\right)$ admits an $\mathscr{A}$-completion.

(d) The set $\mathscr{M}_{0}(\pi, \mathscr{A})$ of pricing probability charges satisfies the condition

$$
\sup _{m \in \mathscr{M}_{0}(\pi, \mathscr{A})} \int(f \wedge 1) d m>0 \quad \text { for all } f \in \mathfrak{F}(\Omega, \mathscr{A}) \text { such that } f>_{*} 0 .
$$

Proof The implication (a) $\Rightarrow$ (b) is immediate in view of the fact that $\tau(\pi)$ is a topology weaker than the topology of uniform distance. Next, assume that (4.1) holds and define the functional

$$
\rho(f)=\inf \left\{\lambda \pi(X)-a: a \in \mathbb{R}, \lambda>0, X \in \mathscr{X} \text { such that } \lambda X \geq_{*} f+a\right\}
$$

for all $f \in L(\mathscr{X}, \mathscr{A})$. Property (2.2) together with $\geq_{*}$-monotonicity of $\pi$ implies that $\rho \in \operatorname{Ext}_{0}(\pi, \mathscr{A})$. Moreover, it is easily seen that

$$
\rho(f+x)=\rho(f)+x, \quad f \in L(\mathscr{X}, \mathscr{A}), x \in \mathbb{R},
$$

and thus that $\rho$ is cash-additive. To prove that $\rho \in \Pi(L(\mathscr{X}, \mathscr{A}))$, fix $f \in L(\mathscr{X}, \mathscr{A})$ such that $f_{1}=f \wedge 1>_{*} 0$. In search of a contradiction, suppose that $\rho\left(f_{1}\right) \leq 0$. Then for each $n \in \mathbb{N}$, there exist $a_{n} \in \mathbb{R}, \lambda_{n}>0$ and $X_{n} \in \mathscr{X}$ such that $\lambda_{n} X_{n} \geq_{*} f_{1}+a_{n}$, but $\lambda_{n} \pi\left(X_{n}\right)<2^{-n}+a_{n}$. This clearly implies

$$
\lambda_{n}\left(X_{n}-\pi\left(X_{n}\right)\right) \geq_{*} f_{1}-2^{-n}
$$


and $f_{1} \in \overline{\mathscr{C}(\pi, \mathscr{A})}^{u}$, contradicting (4.1). It follows that $\rho\left(f_{1}\right)>0$ and hence (b) $\Rightarrow$ (c).

For $(\mathrm{c}) \Rightarrow(\mathrm{d})$, choose $\rho \in \operatorname{Ext}(\pi, \mathscr{A})$ and let $f_{1}$ be defined as above. Consider the linear functional

$$
\hat{\phi}\left(x+b f_{1}\right)=x+b \rho\left(f_{1}\right), \quad x, b \in \mathbb{R},
$$

defined on the linear subspace $L_{0} \subseteq L(\mathscr{X}, \mathscr{A})$ spanned by $\left\{1, f_{1}\right\}$. Given that $\rho$ by its definition satisfies (4.3), $\hat{\phi}$ is dominated by $\rho$ on $L_{0}$ so that we can find an extension $\phi$ of $\hat{\phi}$ to the whole of $L(\mathscr{X}, \mathscr{A})$ still dominated by $\rho$. As in Theorem 3.1, given that $\phi$ is a positive linear functional on a vector lattice of functions, we obtain the representation (A.1) with $m_{\phi} \in \mathscr{M}_{0}(\rho, \mathscr{A}) \subseteq \mathscr{M}_{0}(\pi, \mathscr{A})$. Observe that $f_{1} \sim_{*} f_{1}^{+}$ and that $f_{1}^{+} \in \mathfrak{B}(\Omega, \mathscr{A})$. We deduce from Theorem 3.1 that

$$
0<\rho\left(f_{1}\right)=\phi\left(f_{1}\right)=\phi\left(f_{1}^{+}\right)=\int f_{1}^{+} d m_{\phi}=\int f_{1} d m_{\phi} .
$$

Thus we get $(\mathrm{c}) \Rightarrow(\mathrm{d})$.

Let now $f \in \overline{\mathscr{C}(\pi, \mathscr{A})}^{\tau(\pi)}$. For each $m \in \mathscr{M}_{0}(\pi, \mathscr{A})$ and $n \in \mathbb{N}$, there exist $\lambda^{n}>0, X^{n} \in \mathscr{X}$ and $h_{n} \in L(\mathscr{X}, \mathscr{A})$ such that $\lambda^{n}\left(X^{n}-\pi\left(X^{n}\right)\right) \geq_{*} h_{n}$ and $\int\left(f-h_{n}\right) d m \leq 2^{-n}$. But then

$$
\int f d m \leq 2^{-n}+\lambda^{n}\left(\int X^{n} d m-\pi\left(X^{n}\right)\right) \leq 2^{-n} .
$$

Under (d), this excludes that $f>_{*} 0$ and proves the implication $(\mathrm{d}) \Rightarrow$ (a).

It is immediate to recognise the close relationship linking condition (4.1) to the no-free-lunch-with-vanishing-risk principle formulated long ago by Delbaen and Schachermayer [18] in a highly influential paper. Since then, this condition has been widely accepted as a very convenient mathematical restatement of the no-arbitrage principle. Leaving aside the dissimilarity in the adopted setup, the major difference between (4.1) and NFLVR lies in the interpretation. In fact, due to the restrictions on trade considered here, the elements of the form $X-\pi(X)$ with $X \in \mathscr{X}$ need not correspond to the payoff of any feasible trading strategy so that (4.1) cannot be interpreted as a mathematical reformulation of the no-arbitrage principle. Rather, the set $\overline{\mathscr{C}}(\pi, \mathscr{A})^{u}$ represents those potential claims that cannot be assigned a strictly positive price by any extension of the actual price functional. Thus Theorem 4.1 characterises (4.1) as a condition necessary and sufficient for financial markets to admit a no-arbitrage (in the sense of (2.12)) $\mathscr{A}$-completion. Notice that a strictly positive extension may still exist even when (4.1) fails. In this case, however, it cannot be cash-additive.

In light of the discussion following (3.5), condition (4.2) corresponds to a no-purebubble $(N P B)$ condition, although it does not exclude more general bubbles defined as in (3.4). In other words, with complete financial markets, there cannot exist pure bubbles, which are instead possible with incomplete markets. The fact that completeness of financial markets may change the structure of asset bubbles has already been noted by Jarrow et al. [26] and Herdegen and Schweizer [25, Sect. 6.4.2]. 
Given the arbitrariness of the $\sigma$-algebra $\mathscr{A}$, one may consider the possibility of further extending the market from $L(\mathscr{X}, \mathscr{A})$ to $L\left(\mathscr{X}^{\prime}, \mathscr{A}^{\prime}\right)$, where $\mathscr{X}^{\prime}=L(\mathscr{X}, \mathscr{A})$ and $\mathscr{A} \subseteq \mathscr{A}^{\prime}$. If $\pi^{\prime} \in \operatorname{Ext}(\pi, \mathscr{A})$, it is then immediate from Theorem 4.1 that $\left(\mathscr{X}^{\prime}, \geq_{*}, \pi^{\prime}\right)$ admits an $\mathscr{A}^{\prime}$-completion if and only if

$$
{\overline{\mathscr{C}\left(\pi^{\prime}, \mathscr{A}^{\prime}\right)}}^{u} \cap\left\{f \in \mathfrak{F}\left(\Omega, \mathscr{A}^{\prime}\right): f>_{*} 0\right\}=\varnothing .
$$

Notice that each $\pi^{\prime \prime} \in \operatorname{Ext}\left(\pi^{\prime}, \mathscr{A}^{\prime}\right)$ satisfies the supermartingale-like inequality that $\pi^{\prime \prime} \mid \mathscr{X}^{\prime} \leq \pi^{\prime}$.

Incidentally, we remark that from the equality $\mathscr{M}_{0}\left(\pi_{0}, \mathscr{A}\right)=\mathscr{M}_{0}\left(\pi_{0}^{a}, \mathscr{A}\right)$, it follows that $\pi_{0} \in \Pi_{0}(\mathscr{X})$ satisfies (4.1) if and only if so does $\pi_{0}^{a}$. In fact, we have the following result.

Lemma 4.2 Let $\pi \in \Pi_{0}(\mathscr{X})$. Then:

(a) $\mathscr{C}(\pi, \mathscr{A}) \subseteq \mathscr{C}\left(\pi^{a}, \mathscr{A}\right) \subseteq \overline{\mathscr{C}}(\pi, \mathscr{A})^{u}$.

(b) For every $X \in \mathscr{X}$, we have $\pi^{a}(X) \leq 0$ if and only if $X \in \overline{\mathscr{C}}(\pi, \mathscr{A})^{u}$. Therefore, $\pi^{a} \in \Pi(\mathscr{X})$ if and only if

$$
\overline{\mathscr{C}(\pi, \mathscr{A})}^{u} \cap\left\{X \in \mathscr{X}: X>_{*} 0\right\}=\varnothing .
$$

Proof (a) For each $X \in \mathscr{X}$, it is obvious that $X-\pi(X) \leq X-\pi^{a}(X)$. However, $X-\pi^{a}(X)$ is the uniform limit, as $t \rightarrow \infty$, of $X+t-\pi(X+t) \in \mathscr{C}(\pi, \mathscr{A})$.

(b) $X \in \mathscr{X}$ and $\pi^{a}(X) \leq 0$ imply that for each $n \in \mathbb{N}$ and for $t_{n}>0$ sufficiently large,

$$
X \leq X-\pi^{a}(X) \leq 2^{-n}+\left(X+t_{n}-\pi\left(X+t_{n}\right)\right)
$$

so that $X \in \overline{\mathscr{C}(\pi, \mathscr{A})}^{u}$. Conversely, if $X \leq 2^{-n}+\lambda_{n}\left(X_{n}-\pi\left(X_{n}\right)\right)$ for some $X_{n} \in \mathscr{X}$ and $\lambda_{n} \geq 0$, then, moving $\lambda_{n} \pi\left(X_{n}\right)$ to the left-hand side if positive and using cashadditivity, we conclude that $\pi^{a}(X) \leq 2^{-n}+\lambda_{n}\left(\pi^{a}\left(X_{n}\right)-\pi\left(X_{n}\right)\right) \leq 2^{-n}$.

To highlight the role of competition in financial markets, consider two pricing functionals $\pi, \pi^{\prime} \in \Pi(\mathscr{X})$. If $\pi \leq \pi^{\prime}$, then $\mathscr{C}\left(\pi^{\prime}, \mathscr{A}\right) \subseteq \mathscr{C}(\pi, \mathscr{A})$. Thus lower financial prices are less likely to satisfy (4.1) and thus to admit an extension to a complete financial market free of arbitrage. Competition among market makers, producing lower spreads, may thus have two contrasting effects on economic welfare. On the one hand, it reduces the well-known deadweight loss implicit in monopolistic pricing, while, on the other hand, it imposes a limitation to financial innovation and its benefits in terms of the optimal allocation of risk. It may be conjectured that fully competitive pricing, i.e., the pricing of assets by their fundamental value, may be not compatible with the extension property discussed here. We investigate this issue in Sect. 6 .

\section{Viability}

Following Harrison and Kreps [24] and Kreps [28], several authors in financial economics have characterised the notion of viability, i.e., the property that price func- 
tionals support optimal choice by individuals with monotonic, convex and continuous preferences. Continuity of preferences is a key property in proving the existence of economic equilibrium (see the survey by Mas-Colell and Zame [31]). In addition, in most models, this property provides the necessary justification for formulating the separating condition (4.1) in terms of the closure of $\mathscr{C}(\pi, \mathscr{A})$.

In our model, viability induces a conclusion much stronger than condition (4.1). This latter condition is in fact equivalent to the property that prices be locally viable, a weaker property that will be defined explicitly below. Moreover, the considered restrictions on trade in this work, particularly the constraint that prevents shorting the numéraire, introduce additional difficulties with respect to the approach followed by other papers in this literature (however, see Jouini and Kallal [27] for assumptions on trading restrictions similar to ours).

Consider the space $\mathbb{X}=\mathbb{R} \times \mathfrak{F}(\Omega, \mathscr{A})$, endowed with the topology of uniform convergence, as a description of agents' consumption space at two different instants of time. Agents with no initial endowment are described by a strict preference $\succ$ (i.e., a transitive and nonreflexive binary relation) which induces a family of preferred sets

$$
W_{\succ}(c, h)=\left\{\left(c^{\prime}, h^{\prime}\right) \in \mathbb{X}:\left(c^{\prime}, h^{\prime}\right) \succ(c, h)\right\}, \quad(c, h) \in \mathbb{X},
$$

and by the budget set, defined as

$$
\mathcal{B}(\pi)=\left\{(c, h) \in \mathbb{X}: c+\lambda \pi(X) \leq 0 \text { and } \lambda X \geq_{*} h \text { for some } \lambda>0 \text { and } X \in \mathscr{X}\right\} .
$$

We can then define viability in formal terms.

Definition 5.1 The price functional $\pi$ is viable relatively to the strict preference relation $\succ$ if

$$
\mathcal{B}(\pi) \cap W_{\succ}(0,0)=\varnothing .
$$

Viability has special importance when preferences are monotonic, convex and continuous. In order to define these properties, we extend $>_{*}$ to $\mathbb{X}$ by writing

$$
(c, h)>_{*} 0 \quad \text { whenever } \quad c \geq 0, h \geq_{*} 0 \text { and } c+h>_{*} 0,
$$

and define the convex cone (with the origin deleted)

$$
\mathcal{K}=\left\{(c, h) \in \mathbb{X}:(c, h)>_{*} 0\right\} .
$$

The cone $\mathcal{K}$ plays here the same role as in Kreps [28]. We define now the class of admissible preferences. Let $\operatorname{Int}(A)$ denote the interior of the set $A$. Recall that $\mathbb{X}$ is endowed with the uniform topology.

Definition 5.2 A strict preference $\succ$ on $\mathbb{R} \times \mathfrak{F}(\Omega, \mathscr{A})$ is of class $\mathbf{A}$ if it satisfies the following properties:

$$
\begin{aligned}
& (c, h)+\mathcal{K} \subseteq W_{\succ}(c, h), \quad(c, h) \in \mathbb{X}, \\
& W_{\succ}(c, h) \text { is convex, } \quad(c, h) \in \mathbb{X},
\end{aligned}
$$

for all $k \in \mathcal{K}$, there exists $a_{k}>0$ such that $\left\{a k: a>a_{k}\right\} \subseteq \operatorname{Int}\left(W_{\succ}(0,0)\right)$. 
Condition (5.2) is a monotonicity property, while (5.4) is a weak form of lower semicontinuity. We can now define two distinct notions of viability as follows.

Definition 5.3 The price functional $\pi$ is viable if it is $\succ$-viable for some $\succ$ of class $\mathbf{A}$, and $*$-viable if either

(i) it is viable and $(\varepsilon,-\varepsilon) \in \mathcal{B}(\pi)$ for some $\varepsilon>0$, or

(ii) it is $\succ$-viable for some $\succ$ of class $\mathbf{A}$ which satisfies the additional condition

$$
W_{\succ}(-t, t)=W_{\succ}(0,0) \quad \text { for some } t>0 \text {. }
$$

The literature has rarely considered the need to reinforce the notion of viability as we do here by introducing $*$-viability. The reason is that the two definitions coincide whenever either the numéraire is traded freely or preferences are defined over net trades, and a combination of either one of these two features appears in virtually all contributions. It is thus of some importance to remark that outside of the traditional approach, viability may need to be reformulated.

Another new feature of our model is the focus on a local version of the above properties. If $k \in \mathcal{K}$, a strict preference is of class $\mathbf{A}_{k}$ if it satisfies the above properties (5.2)-(5.4) in restriction to the convex cone $\left\{a k+(b, 0): a, b \in \mathbb{R}_{+}\right\}$(rather than the whole of $\mathcal{K}$ ). If for every $k \in \mathcal{K}$, the price functional $\pi$ is viable (resp. $*$-viable) for some preference of class $\mathbf{A}_{k}$, we speak of $\pi$ as locally viable (resp. locally $*$-viable).

Theorem 5.4 (i) A price functional $\pi$ satisfies (4.1) if and only if it is locally $*$-viable.

(ii) A price functional $\pi$ is $*$-viable if and only if it admits $m \in \mathscr{M}_{0}(\pi, \mathscr{A})$ such that

$$
\int(f \wedge 1) d m>0, \quad f \in L(\mathscr{X}, \mathscr{A}), f>_{*} 0 .
$$

Proof (i) Endow the space $\mathbb{X}_{b}=\mathbb{R} \times \mathfrak{B}(\Omega, \mathscr{A})$ with its associated product norm $\|(c, h)\|=|c|+\|h\|_{\mathfrak{B}(\Omega, \mathscr{A})}$ and assume that $\pi$ is locally $*$-viable. Fix $f>_{*} 0$, write $k=(0, f \wedge 1)$ and choose $\succ$ to be of class $\mathbf{A}_{k}$ and such that $\pi$ is $\succ$-viable. Then $W_{\succ}(0,0) \cap \mathbb{X}_{b}$ and $\mathcal{B}(\pi) \cap \mathbb{X}_{b}$ are disjoint convex sets, the former has nonempty interior by (5.4) and contains a convex cone by (5.2), while the latter contains the origin. There exists then by Dunford and Schwartz [20, V.1.12] a nontrivial continuous linear functional $\Phi$ such that

$$
\inf _{w \in W_{\succ}(0,0) \cap \mathbb{X}_{b}} \Phi(w) \geq 0 \geq \sup _{b \in \mathcal{B}(\pi) \cap \mathbb{X}_{b}} \Phi(b) .
$$

Thus $\Phi((-1,1)) \leq 0$. If $(\varepsilon,-\varepsilon) \in \mathcal{B}(\pi)$ for some $\varepsilon>0$, then $\Phi((-1,1))=0$. Otherwise, $*$-viability implies that $\succ$ may be chosen so as to satisfy (5.5). Fix then $t$ as in (5.5) and let $c>0$ be arbitrary. We have $(c-t, t) \succ(-t, t)$ by (5.2) so that $(c-t, t) \in W_{\succ}(0,0)$. The continuity of $\Phi$ implies that $\Phi((-t, t)) \geq 0$ and again we obtain $\Phi((-1,1))=0$. Given that $\Phi\left(\operatorname{Int}\left(W_{\succ}(0,0)\right)\right)$ is an open interval in $\mathbb{R}_{+}$(see Conway [16, Exercise 3.7]) and that $a k \in \operatorname{Int}\left(W_{\succ}(0,0)\right)$ for some $a>0$, we conclude that $\Phi(k)>0$. By the Riesz representation of continuous linear functionals on 
the space of bounded functions (Dunford and Schwartz [20, IV.5.1]), we conclude that $\Phi$ may be written in the form

$$
\Phi(c, h)=x c+\int h d \mu
$$

for some $x \in \mathbb{R}$ and some charge $\mu \in b a(\mathscr{A})$. The inclusion $-\mathbb{X}_{b,+} \subseteq \mathcal{B}(\pi)$ implies that $x \geq 0, \mu \geq 0$ and $\mu \neq 0$ (because $\left.\Phi(k)=\int(f \wedge 1) d \mu>0\right)$; in addition, $\Phi(1,-1)=0$ leads to $x=\|\mu\|$. By normalisation, we obtain that

$$
\Phi(c, h)=\int(c+h) d m, \quad(c, h) \in \mathbb{X}_{b},
$$

for some $m \in \mathbb{P}_{b a}(\mathscr{A})$. The inclusion $\left(0, \mathbb{1}_{N}\right) \in \mathcal{B}(\pi)$, valid for all $N \in \mathscr{N}_{*}$, implies that $m \in \mathbb{P}_{b a}\left(\mathscr{A}, \mathscr{N}_{*}\right)$. Finally, if $X \in \mathscr{X}$, there exists $a \in \mathbb{R}$ such that $X \sim_{*} X \vee a$ so that for any $n \in \mathbb{N}$, we have the inclusion $\left(-\pi(X),(X \vee a) \wedge 2^{-n}\right) \in \mathcal{B}(\pi) \cap \mathbb{X}_{b}$, from which we deduce that

$$
\begin{aligned}
\int\left(X \wedge 2^{-n}\right) d m & =\int\left((X \vee a) \wedge 2^{-n}\right) d m \\
& =\Phi\left(-\pi(X),(X \vee a) \wedge 2^{-n}\right)+\pi(X) \\
& \leq \pi(X) .
\end{aligned}
$$

This inequality being true for all $X \in \mathscr{X}$ and $n \in \mathbb{N}$ implies via Dunford and Schwartz [20, III.3.6] that $\mathscr{X} \subseteq L^{1}(\Omega, \mathscr{A}, m)$ and finally that $m \in \mathscr{M}_{0}(\pi, \mathscr{A})$. Summing up, for each $f>_{*} 0$, there exists $m \in \mathscr{M}_{0}(\pi, \mathscr{A})$ such that $\int(f \wedge 1) d m>0$, a condition equivalent to (4.1) by Theorem 4.1. This proves the "if" part of (i).

Conversely, assume (4.1), fix $k_{0}=\left(c_{0}, h_{0}\right) \in \mathcal{K}$ and choose $m_{0} \in \mathscr{M}_{0}(\pi, \mathscr{A})$ such that $\int\left(c_{0}+h_{0}\right) d m_{0}>0$ (the existence of such an $m_{0}$ is trivial if $c_{0}>0$ and otherwise follows from (4.2)). Define a strict preference $\succ$ by letting $(c, h) \succ\left(c^{\prime}, h^{\prime}\right)$ whenever $h-h^{\prime} \geq_{*} a$ for some $a \in \mathbb{R}$ and

$$
\left(c-c^{\prime}\right)+\lim _{k \rightarrow \infty} \int\left(\left(h-h^{\prime}\right) \wedge 2^{k}\right) d m_{0}>0 .
$$

Properties (5.2)-(5.4) and (5.5) are easily seen to hold true so that $\succ$ is of class $\mathbf{A}_{k_{0}}$. This, being true for all $k_{0} \in \mathcal{K}$, proves that $\pi$ is locally $*$-viable.

(ii) If $\pi$ is $*$-viable, it is necessarily locally $*$-viable. The proof is identical to that of claim (i), with the only difference that $\operatorname{Int}\left(W_{\succ}(0,0)\right)$ now contains appropriate multiples of each $k \in \mathcal{K}$. Thus if $\Phi$ is the continuous linear functional introduced in (5.7), we conclude that $\Phi(k)>0$ for all $k \in \mathcal{K}$. Proceeding exactly as above, we obtain $m \in \mathscr{M}_{0}(\pi, \mathscr{A})$ that satisfies (5.6). Conversely, if $m \in \mathscr{M}_{0}(\pi, \mathscr{A})$ satisfies that property, the strict preference defined in (5.8) is necessarily $*$-viable.

As is clear from the proof, $*$-viability is required to conclude that the set function $m$ obtained by normalising $\Phi$ is a probability charge. Assuming simple viability, we should in fact obtain an element of $b a_{+}(\mathscr{A})$ which is dominated by $\pi$ on $\mathscr{X}$, and this is weaker than (4.1). 
The main finding of Theorem 5.4 is that $*$-viability of financial prices is a much more restrictive condition than (4.1) and is in fact equivalent to the existence of $m \in \mathscr{M}_{0}(\pi, \mathscr{A})$ that satisfies (5.6). This point is discussed at length in the following Sect. 6 where we provide examples in which such set functions need not exist. Kreps [28, Example 3] has documented other examples of a topological vector space admitting no continuous, strictly positive linear functional and in which, as a consequence, absence of arbitrage is a much weaker condition than admissibility, in line with the conclusions of Theorem 5.4 above. In these cases, the general notion of viability as defined above is not useful. Although this may appear at first as a limitation, the next result suggests that in fact it need not be so, provided we enlarge the set of preferences that we consider as admissible.

Theorem 5.5 The price functional $\pi$ satisfies (4.1) if and only if it is viable for some strict preference $\succ$ which satisfies (5.2), (5.4) as well as either

$$
(c, h) \in W_{\succ}(0,0) \quad \text { if and only if } \quad(c-t, h+t) \in W_{\succ}(0,0) \text { for all } t>0 \text {, }
$$

or

$$
(-t, t) \in \mathcal{B}(\pi) \quad \text { for all } t>0 .
$$

Properties (5.2) and (5.4) are unduly restrictive and a local version may be used instead. In other words, we may require that for each $k \in \mathcal{K}$, there exists a strict preference which is of class $\mathbf{A}_{k}$ (except for convexity) and such that $\pi$ is $\succ$-viable.

Proof of Theorem 5.5 Let $\succ$ meet the conditions of the claim and choose $X \in \mathscr{X}$. If $\pi(X) \leq 0$, then $(0, X-\pi(X)) \in \mathcal{B}(\pi)$; on the other hand, if $\pi(X)>0$, the same conclusion follows from either (5.9) or (5.10). It is thus clear that if $\pi$ is $\succ$-viable, then necessarily the sets $\{X-\pi(X): X \in \mathscr{X}\}$ and $\mathcal{H}=\left\{f \in \mathfrak{F}(\Omega, \mathscr{A}):(0, f) \in W_{\succ}(0,0)\right\}$ are disjoint. By (5.2), the same conclusion remains valid upon replacing the first set with $\mathscr{C}(\pi, \mathscr{A})$. For each $f \in \mathfrak{F}(\Omega, \mathscr{A})$ such that $f>_{*} 0$ (and thus $f \in \mathcal{H}$ ), we deduce from (5.4) that $f \notin \overline{\mathscr{C}(\pi, \mathscr{A})}$. This shows that (4.1) holds. Conversely, if $\pi$ satisfies (4.1), then one may define $(c, h) \succ\left(c^{\prime}, h^{\prime}\right)$ simply by letting

$$
\pi(h)+c>\pi\left(h^{\prime}\right)+c^{\prime} .
$$

It is immediate that this is a strict preference and satisfies (5.2), (5.4) as well as (5.9), while it is obvious that $\pi$ is $\succ$-viable.

In summary, (4.1), i.e., local $*$-viability, is indeed a perfectly sensible condition from the point of view of market equilibrium, at least under either (5.9) or (5.10) and as long as we do not insist on convexity of preferences.

\section{Competitive complete markets}

In this section, we investigate the conditions under which the set $\mathscr{M}_{0}(\pi, \mathscr{A})$ contains a strictly positive element, i.e., some $m$ satisfying (5.6). We denote the corresponding 
set with the symbol $\mathscr{M}(\pi, \mathscr{A})$. In Riedel [33], a set function satisfying (5.6) is said to have full support, and the existence of measures with full support follows easily from the assumption that $\Omega$ is a complete, separable metric space and $\mathscr{X}$ consists of continuous functions defined thereon. Notice that for a pricing probability charge to meet (5.6), it is necessary that it be strictly positive on each non-negligible set. This condition is, however, not sufficient. In fact, although $f>_{*} 0$ implies $\{f>0\} \notin \mathscr{N}_{*}$, the fact that $\mathscr{N}_{*}$ is not closed with respect to countable unions does not rule out the possibility that $\{f>\varepsilon\} \in \mathscr{N}_{*}$ for all $\varepsilon>0$ which contradicts (5.6).

By Theorem 5.4, the condition $\mathscr{M}(\pi, \mathscr{A}) \neq \varnothing$ is equivalent to the price functional $\pi$ being *-viable. More importantly, if $m \in \mathscr{M}(\pi, \mathscr{A})$, it becomes possible to price each asset with payoff in $L(\mathscr{X}, \mathscr{A})$ by its fundamental value (computed according to $m$ ), and the corresponding price functional $\rho$ is free of arbitrage and linear. In other words, $\rho$ provides an $\mathscr{A}$-extension of $\pi$ which is fully competitive, in symbols $\rho \in \operatorname{Ext}(\pi, \mathscr{A})$ and $\mathfrak{m}(\rho)=0$ (recall the definition (2.11) of the market power index $\mathfrak{m})$.

This is of course an extreme situation. The question we want to address is rather: Given a market $\left(\mathscr{X}, \geq_{*}, \pi\right)$, is it possible to find an $\mathscr{A}$-completion that permits some degree of competitiveness? In other words, we look for $\rho \in \operatorname{Ext}(\pi, \mathscr{A})$ satisfying the no-full-monopoly (NFM) condition

$$
\mathfrak{m}(\rho)<1 \text {. }
$$

As we shall see, this condition has in fact far-reaching implications.

Theorem 6.1 A market $\left(\mathscr{X}, \geq_{*}, \pi\right)$ satisfies $\mathscr{M}(\pi, \mathscr{A}) \neq \varnothing$ if and only if it admits an NFM $\mathscr{A}$-completion.

Proof For necessity, take $m \in \mathscr{M}(\pi, \mathscr{A})$ and define $\rho \in \operatorname{Ext}_{0}(\pi, \mathscr{A})$ by

$$
\rho(f)=\int f d m, \quad f \in L(\mathscr{X}, \mathscr{A}) .
$$

Then $\rho$ is cash-additive and $\mathfrak{m}(\rho)=0$ by linearity. Conversely, for sufficiency, assume that $\rho \in \operatorname{Ext}(\pi, \mathscr{A})$ satisfies (6.1). For each $n \in \mathbb{N}$, define the sets

$$
\begin{aligned}
& \mathscr{B}_{0}=\left\{b \in \mathfrak{B}\left(\mathscr{X}, \mathscr{A}, \mathscr{N}_{*}\right): 1 \geq_{*} b>_{*} 0\right\}, \\
& \mathscr{B}_{n}=\left\{b \in \mathscr{B}_{0}: \rho(b)>2 /(n(1-\mathfrak{m}(\rho))\},\right.
\end{aligned}
$$

and let $\operatorname{co}\left(\mathscr{B}_{n}\right)$ be the convex hull of $\mathscr{B}_{n}$. Notice that $\mathscr{B}_{0}=\bigcup_{n \in \mathbb{N}} \mathscr{B}_{n}$ because $\rho \in \operatorname{Ext}(\pi, \mathscr{A})$. If $f=\sum_{i=1}^{N} w_{i} b_{i} \in \operatorname{co}\left(\mathscr{B}_{n}\right)$, then

$$
\rho(f) \geq(1-\mathfrak{m}(\rho)) \sum_{i=1}^{N} w_{i} \rho\left(b_{i}\right) \geq 2 / n .
$$


In view of the properties of $\mathscr{M}_{0}(\pi, \mathscr{A})$ proved in Theorem 3.1, we can apply Sion's minimax theorem, see Sion [35, Corollary 3.3], and obtain from (3.2) that

$$
\inf _{f \in \operatorname{co}\left(\mathscr{B}_{n}\right)} \rho(f)=\inf _{f \in \operatorname{co}\left(\mathscr{B}_{n}\right)} \sup _{\mu \in \mathscr{M}_{0}(\rho, \mathscr{A})} \int f d \mu=\sup _{m \in \mathscr{M}_{0}(\rho, \mathscr{A})} \inf _{f \in \operatorname{co}\left(\mathscr{B}_{n}\right)} \int f d \mu .
$$

Therefore, for each $n \in \mathbb{N}$, there exists $\mu_{n} \in \mathscr{M}_{0}(\rho, \mathscr{A})$ that satisfies the inequality $\inf _{f \in \operatorname{co}\left(\mathscr{B}_{n}\right)} \int f d \mu_{n}>1 / n$. If we define $m=\sum_{n=1}^{\infty} 2^{-n} \mu_{n}$, then $m$ is in $\mathscr{M}_{0}(\rho, \mathscr{A}) \subseteq \mathscr{M}_{0}(\pi, \mathscr{A})$ and as a consequence, $L(\mathscr{X}, \mathscr{A}) \subseteq L^{1}(\Omega, \mathscr{A}, m)$. But then if $f>_{*} 0$, we conclude that $f \wedge 1 \in \mathscr{B}_{0} \subseteq L(\mathscr{X}, \mathscr{A})$, that $f \wedge 1>_{*} 0$ by (2.3), and that $\int(f \wedge 1) d m>0$ so that $m \in \mathscr{M}(\pi, \mathscr{A})$.

What Theorem 6.1 asserts in words is that if an $\mathscr{A}$-complete, arbitrage-free market is possible under limited market power, it is also possible under perfect competition, i.e., with assets priced by their fundamental value. This conclusion does not exclude, however, the somewhat paradoxical situation in which the only possibility to complete the markets is by admitting unlimited market power by financial intermediaries. As noted in the introduction, this situation describes the terms of a potential conflict between the effort of regulating the market power of intermediaries and the support to a process of financial innovation that does not disrupt market stability by introducing arbitrage opportunities.

We stress that the conclusions of Theorem 6.1 crucially depend on the intervening $\sigma$-algebra $\mathscr{A}$ and that the NFM condition is the less likely to hold the finer is $\mathscr{A}$. This suggests that a limited or null market power need not be possible as the degree of complexity of the financial market, as embodied by the size of $\mathscr{A}$, ranks high. Actually, given the requirement $\mathscr{N}_{*} \subseteq \mathscr{A}$, even the smallest possible extension of markets as we defined it need not admit any extension satisfying (6.1).

Consider e.g. the case in which an uncountable family of possible, alternative scenarios is given. In mathematical terms, we can model this situation via an uncountable, pairwise disjoint collection $\left\{A_{\alpha}: \alpha \in \mathfrak{A}\right\}$ of non-negligible sets in $\mathscr{A}$. Then if $\rho \in \operatorname{Ext}(\pi, \mathscr{A})$ and we let $f_{\alpha}=\mathbb{1}_{A_{\alpha}}$, it must be that $\rho\left(f_{\alpha}\right)>0$ for each $\alpha \in \mathfrak{A}$. Thus upon choosing appropriately $\delta>0$ and $\alpha_{1}, \alpha_{2}, \ldots \in \mathfrak{A}$, we obtain

$$
\inf _{n \in \mathbb{N}} \rho\left(f_{\alpha_{n}}\right)>\delta
$$

and consequently $\sum_{1 \leq n \leq N}(1 / N) \rho\left(f_{\alpha_{n}}\right)>\delta$. On the other hand, by disjointness,

$$
\rho\left(\frac{1}{N} \sum_{1 \leq n \leq N} f_{\alpha_{n}}\right) \leq \rho\left(\frac{1}{N} \sup _{1 \leq n \leq N} f_{\alpha_{n}}\right) \leq 1 / N
$$

Thus in the case under consideration, $\mathfrak{m}(\rho)=1$. Under the classical probabilistic assumptions, the existence of the collection $\left\{A_{\alpha}: \alpha \in \mathfrak{A}\right\}$ described above is not possible. (In the theory of Boolean algebras, the condition that no uncountable, pairwise disjoint collection of nonempty sets may be given is known as the countable chain (CC) condition and was first formulated by Maharam [29]. See the comments in Cassese [15]).

A stronger form of the preceding example can be proved. 
Lemma 6.2 If the $\sigma$-algebra $\mathscr{A}$ admits an uncountable collection of non-negligible sets such that the intersection of any two of them is negligible, then any extension $\rho \in \operatorname{Ext}(\pi, \mathscr{A})$ is necessarily such that $\mathfrak{m}(\rho)=1$.

Proof Using the same notation as in the example above, we obtain $\rho\left(f_{\alpha_{n}}\right)>\delta$ as before, while $f_{\alpha_{n}} \wedge f_{\alpha_{m}} \sim_{*} 0$ when $n \neq m$. We obtain

$$
f_{\alpha_{1}} \wedge \sum_{n=2}^{N} f_{\alpha_{n}} \leq\left(f_{\alpha_{1}} \wedge \sum_{n=3}^{N} f_{\alpha_{n}}\right)+\left(f_{\alpha_{1}} \wedge f_{\alpha_{2}}\right) \sim_{*} f_{\alpha_{1}} \wedge \sum_{n=3}^{N} f_{\alpha_{n}}
$$

i.e., by iterating this procedure, $f_{\alpha_{1}} \wedge \sum_{n=2}^{N} f_{\alpha_{n}} \sim_{*}\left(f_{\alpha_{1}} \wedge f_{\alpha_{N}}\right) \sim_{*} 0$. But then

$$
\sum_{n=1}^{N} f_{\alpha_{n}} \sim_{*} f_{\alpha_{1}} \vee \sum_{n=2}^{N} f_{\alpha_{n}} \sim_{*} f_{\alpha_{1}} \vee f_{\alpha_{2}} \vee \sum_{n=3}^{N} f_{\alpha_{n}} \sim_{*} \cdots \sim_{*} \bigvee_{n=1}^{N} f_{\alpha_{n}} \leq 1
$$

Therefore $\rho\left(\frac{1}{N} \sum_{1 \leq n \leq N} f_{\alpha_{n}}\right) \leq \frac{1}{N}$, while $\frac{1}{N} \sum_{1 \leq n \leq N} \rho\left(f_{\alpha_{n}}\right) \geq \delta$.

Lemma 6.2 shows that the complete and competitive financial market imagined by Arrow [4] may be not feasible in a complex world, that is, with a large enough $\mathscr{A}$. We have thus an instance in which complexity acts as a restriction to perfect competition. The only theoretical contribution to the study of the link between competitive equilibria and complexity of which I am aware is the paper of Gale and Sabourian [23] and the references therein. The authors prove that in a game-theoretic matching model, rational agents with aversion to complexity end up playing a subgame-perfect equilibrium which is perfectly competitive despite the finite number of players. Complexity refers here to strategies, and one strategy is considered to be more complex than another whenever the two coincide except on a set of states on which the former is constant.

Lemma 6.2 also provides a negative answer to the question of the existence of viable price systems as defined in Sect. 5. Essentially, this occurs because in the considered situation, the elements of the positive cone $\mathcal{K}$ defined in (5.1) are too numerous and too diverse from one another.

Example 6.3 Let us return to Example 2.2. Let each agent $\alpha$ in the economy be endowed with a probability prior $P_{\alpha}$, forming the collection $\mathscr{P} \subseteq \mathbb{P}(\mathscr{A})$ (thus countably additive). Define $\geq \mathscr{P}$ as in (2.5). If $\mathscr{P}$ is undominated, then by Cassese [12, Theorem 3], there exists an uncountable, pairwise disjoint family of sets in $\mathscr{A}$ each of which is non-negligible for some agent $\alpha$ and thus for the economy as a whole. Denote the indicators of these sets by $f_{\alpha}$. The convex cone $\mathcal{K}$ contains the collection $\mathcal{F}=\left\{f_{\alpha}: \alpha \in \mathfrak{A}\right\}$. By Lemma 6.2 and Theorem 5.4, we know that $\mathscr{M}(\pi, \mathscr{A})=\varnothing$ and as a consequence that $\pi$ is not $*$-viable. Nevertheless, no agent $\alpha$ will consider the entire set $\mathcal{K}$ as relevant for his choices. In fact, most elements of $\mathcal{F}$ are negligible from his point of view, i.e., relatively to $P_{\alpha}$. In other words, no agent respects the condition (5.2) (and a fortiori (5.4)) relatively to $\mathcal{K}$. Nevertheless, for each $\alpha \in \mathfrak{A}$, there is a preference system of class $\mathbf{A}_{f_{\alpha}}$, and thus it is certainly true that an equilibrium-supporting price $\pi$ must be locally $*$-viable, although it cannot be linear (i.e., competitive). 
Those extensions that satisfy the NFM condition have further, special mathematical properties.

Theorem 6.4 Let $\rho \in \Pi(L(\mathscr{X}, \mathscr{A}))$ satisfy (6.1). Then there exists $\mu \in \mathscr{M}(\rho, \mathscr{A})$ such that

$$
\lim _{\mu(|f|) \rightarrow 0} \rho(|f| \wedge 1)=0
$$

Proof Of course, $|f| \wedge 1 \in \mathfrak{B}\left(\Omega, \mathscr{A}, \mathscr{N}_{*}\right)$ for each $f \in \mathfrak{F}(\Omega, \mathscr{A})$ so that (6.2) is well defined. For each $\alpha$ in a given set $\mathfrak{A}$, let $\left\langle A_{n}^{\alpha}\right\rangle_{n \in \mathbb{N}}$ be a decreasing sequence of sets in $\mathscr{A}$ satisfying the following properties: (i) for each distinct pair $\alpha, \beta \in \mathfrak{A}$, there exists $n(\alpha, \beta) \in \mathbb{N}$ such that

$$
A_{n}^{\alpha} \cap A_{n}^{\beta}=\varnothing, \quad n>n(\alpha, \beta),
$$

and (ii) for each $\alpha \in \mathfrak{A}$, there exists $m_{\alpha} \in \mathscr{M}_{0}(\rho, \mathscr{A})$ such that $\lim _{n \rightarrow \infty} m_{\alpha}\left[A_{n}^{\alpha}\right]>0$. If the set $\mathfrak{A}$ is uncountable, then as in Lemma 6.2, we can fix $\delta>0$ and extract a sequence $\alpha_{1}, \alpha_{2}, \ldots$ in $\mathfrak{A}$ such that, letting $f_{n}^{i}=\mathbb{1}_{A_{n}^{\alpha_{i}}}$,

$$
\inf _{i \in \mathbb{N}} \lim _{n \rightarrow \infty} \rho\left(f_{n}^{i}\right)>\delta .
$$

For each $k \in \mathbb{N}$, define $n(k)=1+\sup _{\{i, j \leq k: i \neq j\}} n\left(\alpha_{i}, \alpha_{j}\right)$. Then we conclude that the functions $f_{n(k)}^{1}, \ldots, f_{n(k)}^{k} \in \mathfrak{B}(\Omega, \mathscr{A})$ take values in $[0,1]$, satisfy $f_{n(k)}^{i} \wedge f_{n(k)}^{j}=0$ when $i \neq j$ and

$$
\inf _{1 \leq i \leq k} \rho\left(f_{n(k)}^{i}\right)>\delta
$$

But then, taking $w_{i}=1 / k$, we obtain

$$
\sum_{i=1}^{k} \rho\left(w_{i} f_{n(k)}^{i}\right)>\delta, \quad \text { while } \rho\left(\sum_{i=1}^{k} w_{i} f_{n(k)}^{i}\right)=\frac{1}{k} \rho\left(\sum_{i=1}^{k} f_{n(k)}^{i}\right) \leq \frac{1}{k},
$$

so that $\mathfrak{m}(\rho)=1$, contradicting our initial assumption. We thus reach the conclusion that $\mathfrak{A}$ must be countable and deduce from this and from Cassese [15, Theorem 2] that $\mathscr{M}_{0}(\rho, \mathscr{A})$ is dominated by some of its elements; let this be $\mu$. In addition, $\mathscr{M}_{0}(\rho, \mathscr{A})$ is weak*-compact as a subset of $b a(\mathscr{A})$, as proved in Theorem 3.1. It follows from Zhang [37, Theorem 1.3] that $\mathscr{M}_{0}(\rho, \mathscr{A})$ is weakly compact.

Take a sequence $\left\langle E_{n}\right\rangle_{n \in \mathbb{N}}$ in $\mathscr{A}$ such that $\mu\left[E_{n}\right] \leq 2^{-n}$, and for each $n \in \mathbb{N}$, choose $m_{n} \in \mathscr{M}_{0}(\rho, \mathscr{A})$ such that

$$
m_{n}\left[E_{n}\right]=\sup _{m \in \mathscr{M}_{0}(\pi, \mathscr{A})} m\left[E_{n}\right]
$$

Passing to a subsequence if required, we can assume by virtue of the EberleinŠmulian theorem, see Dunford and Schwartz [20, V.6.1], that $\left\langle m_{n}\right\rangle_{n \in \mathbb{N}}$ is weakly convergent and so, by the finitely additive version of the theorem of Vitali et al. (see 
e.g. Bhaskara Rao and Bhaskara Rao [6, Theorem 8.7.4]), that the set $\left\{m_{n}: n \in \mathbb{N}\right\}$ is uniformly absolutely continuous with respect to $m_{0}=\sum_{n \in \mathbb{N}} 2^{-n} m_{n}$, i.e., that we have $\lim _{m[A] \rightarrow 0} \sup _{n \in \mathbb{N}} m_{n}[A]=0$. However, given that $m_{0} \in \mathscr{M}_{0}(\pi, \mathscr{A})$, we also have $\mu \gg m_{0}$ so that $\left\{m_{n}: n \in \mathbb{N}\right\}$ is uniformly absolutely continuous with respect to $\mu$ as well. Thus

$$
\lim _{\mu[A] \rightarrow 0} \sup _{m \in \mathscr{M}_{0}(\rho, \mathscr{A})} m[A]=0 .
$$

Let $\left\langle f_{n}\right\rangle_{n \in \mathbb{N}}$ be a sequence in $L(\mathscr{X}, \mathscr{A})$ that converges to 0 in $L^{1}(\Omega, \mathscr{A}, \mu)$ and therefore in $\mu$-measure. Then by (3.2) and for arbitrary $0<c<1$,

$$
\lim _{n \rightarrow \infty} \rho\left(\left|f_{n}\right| \wedge 1\right) \leq \lim _{n \rightarrow \infty} \rho\left(\mathbb{1}_{\left\{\left|f_{n}\right|>c\right\}}\right)+c=\lim _{n \rightarrow \infty} \sup _{m \in \mathscr{M}_{0}(\rho, \mathscr{A})} m\left[\left|f_{n}\right|>c\right]+c
$$

so that the claim follows.

To highlight the importance of Theorem 6.1, we note that the absolute continuity property for charges, even if defined on a $\sigma$-algebra, cannot be simply stated in terms of nullsets, but must be defined in terms of convergence to 0 , as the two definitions are no longer equivalent under finite additivity; see Bhaskara Rao and Bhaskara Rao [6, Definition 6.1.1 and Remark 6.1.2]. As a consequence, the existence of a strictly positive element of $\mathscr{M}_{0}(\pi, \mathscr{A})$ established in Theorem 6.1 is not sufficient to imply the existence of a probability charge $\mu$ with respect to which each element of $\mathscr{M}_{0}(\pi, \mathscr{A})$ is absolutely continuous. It rather induces the weaker conclusion that there is a pricing measure $m_{0}$ such that $m_{0}[A]=0$ implies $m[A]=0$ for all $m \in \mathscr{M}_{0}(\pi, \mathscr{A})$.

On the other hand, if there exists a probability charge $\mu$ such that $m \ll \mu$ for each $m \in \mathscr{M}_{0}(\pi, \mathscr{A})$, then $\mathscr{M}_{0}(\pi, \mathscr{A})$ is uniformly absolutely continuous with respect to $\mu$, since as seen in the proof of Theorem $6.4, \mathscr{M}_{0}(\pi, \mathscr{A})$ is weakly compact. A similar conclusion is not true in the countably additive case treated in the traditional approach. In that approach, risk neutral measures are absolutely continuous with respect to the given reference probability measure by assumption, but the set of risk neutral measures is not weak*-compact when regarded as a subset of ba( $\mathscr{A})$. This special feature illustrates a possible advantage of the finitely additive approach over the countably additive one.

Finally, notice that Theorem 6.4 does not require the no-arbitrage property (in the sense of (2.12)) and may thus be adapted to the case in which $L(\mathscr{X}, \mathscr{A})$ is a generic vector lattice of functions on $\Omega$ containing the bounded functions and $\rho$ is a monotonic, subadditive and cash-additive functional, such as the Choquet integral with respect to a submodular capacity.

Another characterisation of the condition $\mathscr{M}(\pi, \mathscr{A}) \neq \varnothing$ may be obtained as follows.

Theorem 6.5 A market $\left(\mathscr{X}, \geq_{*}, \pi\right)$ satisfies the condition $\mathscr{M}(\pi, \mathscr{A}) \neq \varnothing$ if and only if there exists $P \in \mathbb{P}_{b a}\left(\mathscr{A}, \mathscr{N}_{*}\right)$ such that

$$
\begin{aligned}
& \mathscr{X} \subseteq L^{1}(\Omega, \mathscr{A}, P) \text { and } \\
& \overline{\mathscr{C}(\pi, \mathscr{A})}^{L^{1}(\Omega, \mathscr{A}, P)} \cap\left\{f \in \mathfrak{F}(\Omega, \mathscr{A}): f>_{*} 0\right\}=\varnothing .
\end{aligned}
$$


Conversely, if $P \in \mathbb{P}_{b a}\left(\mathscr{A}, \mathscr{N}_{*}\right)$ satisfies (6.3), then $\mathscr{N}_{*}$ coincides with the collection $\mathscr{N}_{P}$ of $P$-nullsets.

Proof If $\mu \in \mathscr{M}(\pi, \mathscr{A})$, then by definition $\mathscr{X} \subseteq L^{1}(\Omega, \mathscr{A}, \mu)$ and $\int f d \mu \leq 0$ for each $f \in \overline{\mathscr{C}(\pi, \mathscr{A})} L^{1}(\Omega, \mathscr{A}, \mu)$, which rules out $f>_{*} 0$. Choose $P=\mu$. Conversely, if $P \in \mathbb{P}_{b a}\left(\mathscr{A}, \mathscr{N}_{*}\right)$ satisfies (6.3) and $h>_{*} 0$, then $h \wedge 1 \in L^{1}(\Omega, \mathscr{A}, P)$ and $h \wedge 1>_{*} 0$. There exists then a positive and continuous linear functional $\phi_{h}$ on $L^{1}(\Omega, \mathscr{A}, P)$ such that

$$
\sup \left\{\phi_{h}(f): f \in \overline{\mathscr{C}(\pi, \mathscr{A})}^{L^{1}(\Omega, \mathscr{A}, P)}\right\} \leq 0<\phi_{h}(h \wedge 1) .
$$

Given that necessarily $\phi_{h}(1)>0,(6.4)$ remains unchanged if we replace $\phi_{h}$ by its normalisation so that we can assume $\phi_{h}(1)=1$. This implies that $\phi_{h} \in \operatorname{Ext}_{0}(\pi, \mathscr{A})$ and, by Theorem A.3, that $\phi_{h}$ admits the representation

$$
\phi_{h}(f)=\int f d m_{h}, \quad f \in L^{1}(\Omega, \mathscr{A}, \mu),
$$

for some $m_{h} \in \mathscr{M}_{0}(\pi, \mathscr{A})$ that satisfies the inclusion $L^{1}(\Omega, \mathscr{A}, P) \subseteq L^{1}\left(\Omega, \mathscr{A}, m_{h}\right)$ and thus is such that $m_{h} \ll P$. But then, if $h=\mathbb{1}_{A}$ and $A \notin \mathscr{N}_{*}$, we conclude that $P[A]>0$. Moreover, by the finitely additive version of the Halmos and Savage theorem (see Cassese [12, Theorem 1]), the set $\left\{m_{h}: h \in \mathfrak{F}(\Omega, \mathscr{A}), h>_{*} 0\right\}$ is dominated by some $m_{0} \in \mathscr{M}_{0}(\pi, \mathscr{A})$. It is then clear that $\int(f \wedge 1) d m_{0}>0$ for all $f>_{*} 0$ and thus that $m_{0} \in \mathscr{M}(\pi, \mathscr{A})$.

Notice that if $P \in \mathscr{M}(\pi, \mathscr{A})$, then a set is negligible if and only if it is $P$-null and this, as was shown in Sect. 2.1, is necessary and sufficient for the equivalence of the ranking $\geq_{*}$ and the ranking $\geq_{P}$ defined in (2.2). Thus Theorem 6.5 provides an implicit answer to the question of whether the rationality criterion $\geq_{*}$ may be induced by some (endogenous) probability.

\section{Countably additive markets}

Given the emphasis on countable additivity which dominates the traditional financial literature, it is natural to ask if it is possible to characterise those markets in which the set $\mathscr{M}_{0}(\pi, \mathscr{A})$ contains a countably additive element. A more ambitious question is whether such a measure is strictly positive, i.e., an element on $\mathscr{M}(\pi, \mathscr{A})$.

Not surprisingly, an exact characterisation may be obtained by considering the fairly unnatural possibility of forming portfolios which invest simultaneously in countably many different assets. This induces to modify the definition (2.11) into (again with the convention $0 / 0=0$ )

$$
\mathfrak{m}^{c}\left(\rho ; f_{1}, f_{2}, \ldots\right)=\lim _{k \rightarrow \infty} \frac{\sum_{n \leq k} \rho\left(f_{n}\right)-\rho\left(\sum_{n \in \mathbb{N}} f_{n}\right)}{\sum_{n \leq k} \rho\left(f_{n}\right)}, \quad \rho \in \Pi(L(\mathscr{X}, \mathscr{A})),
$$

for all sequences $f_{1}, f_{2}, \ldots \in \mathfrak{B}_{+}(\Omega, \mathscr{A})$ such that $\sum_{n \in \mathbb{N}} f_{n} \in \mathfrak{B}(\Omega, \mathscr{A})$. 
It may at first appear obvious that in the presence of transaction fees, the cost of buying separately each component of a given portfolio is higher than the cost of purchasing the whole portfolio in a single transaction. But considered more carefully, this is indeed correct only if the infinite sum $\sum_{n \in \mathbb{N}} \rho\left(f_{n}\right)$ corresponds to an actual cost, i.e., only if the strategy of buying separately infinitely many assets is feasible in the market.

Define then the functional

$$
\mathfrak{m}^{c}(\rho)=\inf \mathfrak{m}^{c}\left(\rho ; f_{1}, f_{2}, \ldots\right),
$$

where the infimum is computed with respect to all sequences in $\mathfrak{B}_{+}(\Omega, \mathscr{A})$ whose sum is bounded in $\omega$. Notice that in general, the inequality $\mathfrak{m}^{c}\left(\rho ; f_{1}, f_{2}, \ldots\right) \geq 0$ is no longer valid, while of course $\mathfrak{m}^{c}(\rho) \leq \mathfrak{m}(\rho)$.

Theorem 7.1 Let $\pi \in \Pi_{0}(\mathscr{X})$. Then:

(a) $\mathscr{M}_{0}(\pi, \mathscr{A}) \cap \mathbb{P}(\mathscr{A}) \neq \varnothing$ if and only if there exists $\rho \in \operatorname{Ext}_{0}(\pi, \mathscr{A})$ such that $\mathfrak{m}^{c}(\rho)>-\infty$ and

$$
\sum_{n \in \mathbb{N}} \rho\left(f_{n}\right)<\infty \quad \text { for all } f_{1}, f_{2}, \ldots \text { in } \mathfrak{B}_{+}(\Omega, \mathscr{A}) \text { with } \sum_{n \in \mathbb{N}} f_{n} \in \mathfrak{B}(\Omega, \mathscr{A})
$$

(b) $\mathscr{M}(\pi, \mathscr{A}) \cap \mathbb{P}(\mathscr{A}) \neq \varnothing$ if and only if there exists $\rho \in \operatorname{Ext}(\pi, \mathscr{A})$ such that

$$
1>\mathfrak{m}(\rho) \geq \mathfrak{m}^{c}(\rho)>-\infty .
$$

Proof Of course, any element $m \in \mathscr{M}_{0}(\pi, \mathscr{A}) \cap \mathbb{P}(\mathscr{A})$ when considered as a pricing functional is an element of $\operatorname{Ext}_{0}(\pi, \mathscr{A})$ with the property that $\mathfrak{m}^{c}(m)=\mathfrak{m}(m)=0$. This proves necessity for both claims. To prove sufficiency, let $\rho \in \Pi_{0}(L(\mathscr{X}, \mathscr{A}))$ and choose a sequence $\left\langle f_{n}\right\rangle_{n \in \mathbb{N}}$ in $\mathfrak{B}_{+}(\Omega, \mathscr{A})$ with $\sum_{n \in \mathbb{N}} f_{n} \in \mathfrak{B}(\Omega, \mathscr{A})$. If $\mathfrak{m}(\rho)<1$, then

$$
\sum_{n \in \mathbb{N}} \rho\left(f_{n}\right)=\lim _{k \rightarrow \infty} \sum_{n \leq k} \rho\left(f_{n}\right) \leq \lim _{k \rightarrow \infty} \frac{\rho\left(\sum_{n \leq k} f_{n}\right)}{1-\mathfrak{m}(\rho)} \leq \frac{\rho\left(\sum_{n \in \mathbb{N}} f_{n}\right)}{1-\mathfrak{m}(\rho)}<\infty
$$

so that (7.1) is satisfied. It is therefore enough to show that if $\rho$ meets the conditions listed under (a), then $\mathscr{M}_{0}(\rho, \mathscr{A}) \subseteq \mathbb{P}(\mathscr{A})$. Choose $m \in \mathscr{M}_{0}(\rho, \mathscr{A})$, let $\left\langle A_{n}\right\rangle_{n \in \mathbb{N}}$ be a disjoint sequence in $\mathscr{A}$ and let $f_{n}=\mathbb{1}_{A_{n}}$. We get $\sum_{n \in \mathbb{N}} \rho\left(f_{n}\right)<\infty$ and therefore

$$
\begin{aligned}
m\left[\bigcup_{n \in \mathbb{N}} A_{n}\right] & =\sum_{n \leq k} m\left[A_{n}\right]+\lim _{k \rightarrow \infty} m\left[\bigcup_{n>k} A_{n}\right] \\
& \leq \sum_{n \in \mathbb{N}} m\left[A_{n}\right]+\lim _{k \rightarrow \infty} \rho\left(\sum_{n>k} f_{n}\right) \\
& \leq \sum_{n \in \mathbb{N}} m\left[A_{n}\right]+\left(1-\mathfrak{m}^{c}(\rho)\right) \lim _{k \rightarrow \infty} \sum_{n>k} \rho\left(f_{n}\right) \\
& =\sum_{n \in \mathbb{N}} m\left[A_{n}\right] .
\end{aligned}
$$


Thus $\mathscr{M}_{0}(\rho, \mathscr{A}) \subseteq \mathbb{P}(\mathscr{A})$. Claim (b) follows from the preceding remarks and Theorem 6.1 .

The conditions for the existence of a countably additive pricing measure listed under (a) and (b) are perhaps deceptively simple. They are in fact increasingly restrictive, the finer the $\sigma$-algebra $\mathscr{A}$ is. With $\mathscr{A}$ equal to the power set of $\Omega$, virtually every sequence of positive and bounded functions may potentially produce a violation of the condition $\mathfrak{m}^{c}(\rho)>-\infty$. To stress this point, we observe that this inequality implies that whenever $\left\langle f_{n}\right\rangle_{n \in \mathbb{N}}$ is a uniformly bounded sequence of negligible functions, then necessarily $\sup _{n \in \mathbb{N}} f_{n}$ must be negligible as well. An obvious implication is that $\mathscr{N}_{*}$ must be closed with respect to countable unions, a property that represents a significant change of the axioms (2.1)-(2.3) that characterise economic rationality as embodied into $\geq_{*}$. There may well be cases in which the closedness of $\mathscr{N}_{*}$ with respect to countable unions is simply contradictory and which cast doubts on the economic adequacy of the countably additive paradigm. A model in which the sample space $\Omega$ is a separable metric space is a good case in point. In fact, if we take $\mathscr{N}_{*}$ to consist of sets of the first category - which would clearly be a good example of what people consider as negligible -, then as is well known, $\mathbb{P}\left(\mathscr{A}, \mathscr{N}_{*}\right)=\varnothing$; see Szpilrajn-Marczewski [36, Théorème 1].

\section{Appendix A: Auxiliary results}

\section{A.1 The finitely additive integral}

In order for this work to be as self-contained as possible, we recall from Dunford and Schwartz [20, Chap. III.3] and Bhaskara Rao and Bhaskara Rao [6, Chap. 4] some basic facts about the finitely additive integral. Let then $m \in b a_{+}(\mathscr{A})$.

Definition A.1 Function $f \in \mathfrak{F}(\Omega)$ is said to be integrable with respect to $m$ - which we write in symbols as $f \in L^{1}(\Omega, \mathscr{A}, m)$ - if there exists a sequence $\left\langle f_{n}\right\rangle_{n \in \mathbb{N}}$ of $\mathscr{A}$-measurable, simple functions such that each $f_{n}$ is integrable, $f_{n}$ converges to $f$ in $m$-measure and

$$
\lim _{k, n \rightarrow \infty} \int\left|f_{n}-f_{k}\right| d m=0
$$

Theorem A.2 (Dunford and Schwartz [20, III.3.6]) Let $\left\langle h_{n}\right\rangle_{n \in \mathbb{N}}$ be a sequence in $L^{1}(\Omega, \mathscr{A}, m)$ and $h \in \mathfrak{F}(\Omega)$. Then we have that $h \in L^{1}(\Omega, \mathscr{A}, m)$ and $\left(h_{n}\right)$ converges to $h$ in the norm of $L^{1}(\Omega, \mathscr{A}, m)$ if and only if $\left(h_{n}\right)$ converges to $h$ in $m$-measure and $\lim _{m[A] \rightarrow 0} \sup _{n \in \mathbb{N}} \int_{A}\left|h_{n}\right| d m=0$.

\section{A.2 Integral representation}

Because of its repeated use in several proofs in this paper, we restate for ease of reference the following general representation theorem which is just an adaptation of Cassese [14, Theorem 3.3] to the special case considered in this work. 
Theorem A.3 Let L be a vector sublattice of $\mathfrak{F}(\Omega, \mathscr{A})$ and $\phi$ a positive linear functional defined thereon. There exist a positive linear functional $\phi^{\perp}$ on $L$ and a positive set function $\lambda \in b a_{+}(\Omega, \mathscr{A})$ such that $\phi^{\perp}(f)=0$ for all $f \in L \cap \mathfrak{B}(\Omega, \mathscr{A})$, $L \subseteq L^{1}(\Omega, \mathscr{A}, \lambda)$ and

$$
\phi(f)=\phi^{\perp}(f)+\int f d \lambda, \quad f \in L
$$

If $L$ is such that $f \wedge 1 \in L$ for each $f \in L$, then $\phi^{\perp}=0$ if and only if

$$
\phi(f)=\lim _{k \rightarrow \infty} \phi\left(f^{+} \wedge k-f^{-} \wedge k\right), \quad f \in L .
$$

Proof The existence of the representation (A.1) for some $\lambda \in b a_{+}\left(\Omega, 2^{\Omega}\right)$ follows immediately from [14, Theorem 3.3]. Indeed, given that $\phi$ is assumed to be positive, the identity map on $L$ is clearly $\phi$-conglomerative, and since $L$ is a lattice, directed as well. Given that $L$ consists of $\mathscr{A}$-measurable functions, the representing charge $\lambda$ may be restricted to $\mathscr{A}$. Denote that restriction again by $\lambda$. If $f \wedge 1 \in L$ for each $f \in L$, then $f_{k}=f^{+} \wedge k-f^{-} \wedge k \in L$. Since $f \in L^{1}(\Omega, \mathscr{A}, \lambda)$, we obtain from ordinary results on finitely additive integrals, see Dunford and Schwartz [20, III.3.6], that $\lim _{k \rightarrow \infty} \int\left|f-f_{k}\right| d \lambda=0$. Thus if $\phi^{\perp}=0$, then (A.2) holds; conversely, (A.2) implies

$$
\phi^{\perp}(f)=\lim _{k \rightarrow \infty}\left(\phi\left(f_{k}\right)-\int f_{k} d \lambda\right)=\lim _{k \rightarrow \infty} \phi^{\perp}\left(f_{k}\right)=0
$$

Funding Note Open access funding provided by Università degli Studi di Milano - Bicocca within the CRUI-CARE Agreement.

Open Access This article is licensed under a Creative Commons Attribution 4.0 International License, which permits use, sharing, adaptation, distribution and reproduction in any medium or format, as long as you give appropriate credit to the original author(s) and the source, provide a link to the Creative Commons licence, and indicate if changes were made. The images or other third party material in this article are included in the article's Creative Commons licence, unless indicated otherwise in a credit line to the material. If material is not included in the article's Creative Commons licence and your intended use is not permitted by statutory regulation or exceeds the permitted use, you will need to obtain permission directly from the copyright holder. To view a copy of this licence, visit http://creativecommons.org/licenses/by/ $4.0 \%$.

\section{References}

1. Acciaio, B., Beiglböck, M., Penkner, F., Schachermayer, W.: A model-free version of the fundamental theorem of asset pricing and the super-replication theorem. Math. Finance 26, 233-251 (2016)

2. Allen, F., Gale, D.: Arbitrage, short sales, and financial innovation. Econometrica 59, 1041-1068 (1991)

3. Araujo, A., Chateauneuf, A., Faro, J.H.: Pricing rules and Arrow-Debreu ambiguous valuation. Econ. Theory 49, 1-35 (2012)

4. Arrow, K.J.: The rôle of securities in the optimal allocation of risk bearing. Rev. Econ. Stud. 31, 91-96 (1964)

5. Bewley, T.F.: Existence of equilibria in economies with infinitely many commodities. J. Econ. Theory 4, 514-540 (1972) 
6. Bhaskara Rao, K.P.S., Bhaskara Rao, M.: Theory of Charges. Academic Press, London (1983)

7. Biais, B., Glosten, L., Spatt, C.: Market microstructure: a survey of microfoundations, empirical results, and policy implications. J. Financ. Mark. 8, 217-264 (2005)

8. Bisin, A.: General equilibrium with endogenously incomplete financial markets. J. Econ. Theory 82, 19-45 (1998)

9. Bouchard, B., Nutz, M.: Arbitrage and duality in nondominated discrete-time models. Ann. Appl. Probab. 25, 823-859 (2015)

10. Burzoni, M., Riedel, F., Soner, M.: Viability and arbitrage under Knightian uncertainty. Econometrica 89, 1207-1234 (2021)

11. Cassese, G.: Asset pricing with no exogenous probability measure. Math. Finance 18, 23-54 (2008)

12. Cassese, G.: The theorem of Halmos and Savage under finite additivity. J. Math. Anal. Appl. 437, 870-881 (2016)

13. Cassese, G.: Asset pricing in an imperfect world. Econom. Theory 64, 539-570 (2017)

14. Cassese, G.: Conglomerability and the representation of linear functionals. J. Convex Anal. 25, 789-815 (2018)

15. Cassese, G.: Control measures on Boolean algebras. J. Math. Anal. Appl. 478, 764-775 (2019)

16. Conway, J.B.: Course in Functional Analysis. Springer, Berlin (1990)

17. Davis, M.H.A., Hobson, D.G.: The range of traded option prices. Math. Finance 17, 1-14 (2007)

18. Delbaen, F., Schachermayer, W.: A general version of the fundamental theorem of asset pricing. Math. Ann. 300, 463-520 (1994)

19. Dellacherie, C., Meyer, P.-A.: Probabilities and Potential A. North-Holland, Amsterdam (1978)

20. Dunford, N.J., Schwartz, J.T.: Linear Operators. Part I, 2nd edn. Wiley and Sons, New York (1988)

21. El Karoui, N., Ravanelli, C.: Cash subadditive risk measures and interest rate ambiguity. Math. Finance 19, 561-590 (2009)

22. Epstein, L.G., Ji, S.: Ambiguous volatility and asset pricing in continuous time. Rev. Financ. Stud. 26, 1740-1786 (2013)

23. Gale, D., Sabourian, H.: Complexity and competition. Econometrica 73, 739-769 (2005)

24. Harrison, M.J., Kreps, D.M.: Martingales and arbitrage in multiperiod securities markets. J. Econ. Theory 20, 381-408 (1979)

25. Herdegen, M., Schweizer, M.: Strong bubbles and strict local martingales. Int. J. Theor. Appl. Finance 19, 1650022 (2016)

26. Jarrow, R.A., Protter, P., Shimbo, K.: Asset price bubbles in complete markets. In: Fu, M.C., et al. (eds.) Advances in Mathematical Finance. Festschrift for Dilip Madan, pp. 97-121. Birkhäuser, Boston (2007)

27. Jouini, E., Kallal, H.: Viability and equilibrium in securities markets with frictions. Math. Finance 9, 275-292 (1999)

28. Kreps, D.M.: Arbitrage and equilibrium in economies with infinitely many commodities. J. Math. Econ. 8, 15-35 (1981)

29. Maharam, D.: An algebraic characterization of measure algebras. Ann. Math. 48, 154-167 (1947)

30. Mas-Colell, A.: The price equilibrium existence problem in topological vector lattices. Econometrica 54, 1039-1053 (1986)

31. Mas-Colell, A., Zame, W.: Equilibrium theory in infinite dimensional spaces. In: Hildenbrand, W., Sonnenschein, H. (eds.) Handbook of Mathematical Economics, 1st edn. vol. 4, pp. 1835-1898. Elsevier, Amsterdam (1991)

32. Radner, R.: Existence of equilibrium of plans, prices, and price expectations in a sequence of markets. Econometrica 40, 289-303 (1972)

33. Riedel, F.: Finance without probabilistic prior assumptions. Decis. Econ. Finance 38, 75-91 (2015)

34. Schmeidler, D.: Subjective probability and expected utility without additivity. Econometrica 57, 571-587 (1989)

35. Sion, M.: On general minimax theorems. Pac. J. Math. 8, 171-175 (1958)

36. Szpilrajn-Marczewski, E.: Remarques sur les fonctions complètement additives d'ensemble et sur les ensembles jouissant de la propriété de Baire. Fundam. Math. 22, 303-311 (1934)

37. Zhang, X.-D.: On weak compactness in spaces of measures. J. Funct. Anal. 143, 1-9 (1997)

Publisher's Note Springer Nature remains neutral with regard to jurisdictional claims in published maps and institutional affiliations. 